\title{
Application of the Nonlinear Steepest Descent Method to the Coupled Sasa-Satsuma Equation
}

\author{
Xianguo Geng, Mingming Chen* and Kedong Wang \\ School of Mathematics and Statistics, Zhengzhou University, 100 Kexue Road, \\ Zhengzhou, Henan 450001, China.
}

Received 22 September 2020; Accepted (in revised version) 25 September 2020.

\begin{abstract}
We use spectral analysis to reduce Cauchy problem for the coupled SasaSatsuma equation to a $5 \times 5$ matrix Riemann-Hilbert problem. The upper and lower triangular factorisations of the jump matrix and a decomposition of the vector-valued spectral function are given. Applying various transformations related to the RiemannHilbert problems and suitable decompositions of the jump contours and the nonlinear steepest descent method, we establish the long-time asymptotics of the problem.
\end{abstract}

AMS subject classifications: 35Q53, 35B40, 35Q55

Key words: Coupled Sasa-Satsuma equation, nonlinear steepest descent method, long-time asymptotics.

\section{Introduction}

The Sasa-Satsuma equation

$$
u_{t}+u_{x x x}+6|u|^{2} u_{x}+3 u\left(|u|^{2}\right)_{x}=0
$$

also called the higher-order nonlinear Schrödinger equation, was originally aimed to describe the propagation of pulses in optical fiber $[18,19]$. It attracted a considerable attention and has been extensively studied because of significant applications. The inverse scattering method [34] and the Hirota bilinear method [12] were used to obtain $N$-soliton solution of this equation. On the other hand, by linearising the corresponding spectral operator it was shown that the squared eigenfunctions of the Sasa-Satsuma equation can be represented as the sums of two terms, each of which is a product of Jost and adjoint Jost functions [43]. Akhmedieva et al. [2] studied the rogue wave spectra of the Eq. (1.1) and its presence in the spectra of chaotic wave fields produced by the modulation instability. Ling [22] obtained high order solution formulas in the determinant form by using a generalised Darboux transformation and the formal series method. In [44], finite genus solutions

${ }^{*}$ Corresponding author. Email addresses: $\quad$ xggeng@zzu.edu.cn (X.G. Geng), chenmmzzu@163.com (M.M. Chen), wangkdmath@163.com (K.D. Wang) 
of the Sasa-Satsuma hierarchy, associated with a $3 \times 3$ matrix spectral problem, are obtained by using asymptotic expansions of the Baker-Akhiezer function and its Riemann theta function representation [37]. The Riemann-Hilbert approach, Darboux transformation and Riccati equation are employed in investigating the integrability of multi-coupled nonlinear integrable equations and finding their exact solutions - cf. Refs. $[9,11,15,20,21,27,38,41]$.

Let

$$
\mathscr{S}(\mathbb{R})=\left\{f(x) \in C^{\infty}(\mathbb{R}): \sup _{x \in \mathbb{R}}\left|x^{\alpha} \partial^{\beta} f(x)\right|<\infty, \forall \alpha, \beta \in \mathbb{N}\right\}
$$

be the Schwartz class. In this work, we use the nonlinear steepest descent method in order to study the long-time asymptotic behavior of the Cauchy problem for the coupled SasaSatsuma equation

$$
\begin{aligned}
& u_{t}+u_{x x x}+6\left(|u|^{2}+|v|^{2}\right) u_{x}+3 u\left(|u|^{2}+|v|^{2}\right)_{x}=0, \\
& v_{t}+v_{x x x}+6\left(|u|^{2}+|v|^{2}\right) v_{x}+3 v\left(|u|^{2}+|v|^{2}\right)_{x}=0, \\
& u(x, 0)=u_{0}(x), \quad v(x, 0)=v_{0}(x),
\end{aligned}
$$

where $u(x, t)$ and $v(x, t)$ are complex-valued potentials, $u_{0}(x), v_{0}(x) \in \mathscr{S}(\mathbb{R})$ and are generic in the sense that the below defined determinant det $a(k)$ does not vanish in the lower complex half $k$-plane $\mathbb{C}_{-}$. The coupled Sasa-Satsuma equation can describe the simultaneous propagation in birefringent or two-mode fibers [32]. In [40], multi-soliton solutions of the coupled Sasa-Satsuma equation are derived by solving a Riemann-Hilbert problem. Besides, infiniteness of conserved quantities of the Eqs. (1.2) is discussed in [33], the Painlevé property in [36], and some other characteristics in $[24,28,45]$. The Deift-Zhou nonlinear steepest descent method introduced in [7] is aimed to study the long-time asymptotic behavior of solutions for the mKdV equation. The method was subsequently applied to a number of integrable nonlinear evolution equations associated with numerous matrix spectral problems $[4-6,8,10,13,16,17,23,25,26,29-31,35,42]$. However, to the best of author's knowledge, the nonlinear steepest descent method has not been used in the study of long-time asymptotics for integrable equation associated with $5 \times 5$ matrix Lax pairs and the aim of this work is to extend the Deift-Zhou method to the Eqs. (1.2) associated with such Lax pairs. The main result of this paper is the following theorem.

Theorem 1.1. Let $(u(x, t), v(x, t))$ be the solution for the Cauchy problem of the coupled Sasa-Satsuma equation (1.2) with $u_{0}(x)$ and $v_{0}(x) \in \mathscr{S}(\mathbb{R})$. If $x<0$ and $|x / t| \leq C$, as $t \rightarrow \infty$, then the leading asymptotics of $(u(x, t), v(x, t))$ has the form

$$
\begin{aligned}
& (u(x, t), v(x, t)) \\
= & -\frac{v e^{\pi v / 2}}{\sqrt{24 t k_{0} \pi}}\left[\delta_{A}^{2} e^{-\pi i / 4} \Gamma(-i v)\left(\gamma_{2}\left(k_{0}\right), \gamma_{4}\left(k_{0}\right)\right)+\delta_{A}^{-2} e^{\pi i / 4} \Gamma(i v)\left(\gamma_{1}^{*}\left(k_{0}\right), \gamma_{3}^{*}\left(k_{0}\right)\right)\right] \\
+ & \mathscr{O}\left(c\left(k_{0}\right) t^{-1} \log t\right),
\end{aligned}
$$

where $C$ is a constant, $\Gamma$ the Gamma function, $\gamma(k)=\left(\gamma_{1}(k), \gamma_{2}(k), \gamma_{3}(k), \gamma_{4}(k)\right)$ the vector- 
valued function defined in (2.7), $c$ a rapidly decreasing function, and

$$
\begin{aligned}
& \delta_{A}=e^{\chi\left(-k_{0}\right)-8 i t k_{0}^{3}}\left(192 t k_{0}^{3}\right)^{i v / 2}, \\
& k_{0}=\sqrt{\frac{-x}{12 t}}, \quad v=-\frac{1}{2 \pi} \log \left(1+\left|\gamma\left(k_{0}\right)\right|^{2}\right), \\
& \chi\left(-k_{0}\right)=\frac{1}{2 \pi i} \int_{-k_{0}}^{k_{0}} \log \left(\frac{1+|\gamma(\xi)|^{2}}{1+\left|\gamma\left(k_{0}\right)\right|^{2}}\right) \frac{d \xi}{\xi+k_{0}} .
\end{aligned}
$$

The outline of this paper is as follows. In Section 2, the inverse scattering method is used to transform the Cauchy problem for the coupled Sasa-Satsuma equation into a matrix Riemann-Hilbert problem. In Section 3, the original matrix Riemann-Hilbert problem is reduced to a model Riemann-Hilbert problem whose solution can be represented via parabolic cylinder functions. Finally, we obtain the long-time asymptotics of the Cauchy problem for the coupled Sasa-Satsuma equation.

\section{Inverse Scattering Method and Riemann-Hilbert Problems}

The Eq. (1.2) is the compatibility condition for the Lax pair

$$
\begin{aligned}
& \psi_{x}=(-i k \sigma+U) \psi, \\
& \psi_{t}=\left(-4 i k^{3} \sigma+Q\right) \psi,
\end{aligned}
$$

where $\psi=\psi(k ; x, t)$ is a $5 \times 5$ matrix, $\sigma=\operatorname{diag}\{1,1,1,1,-1\}$, and

$$
\begin{aligned}
& U=\left(\begin{array}{ll}
0_{4 \times 4} & q \\
-q^{\dagger} & 0
\end{array}\right), \quad q=\left(u, u^{*}, v, v^{*}\right)^{\mathrm{T}}, \\
& Q=4 k^{2} U+2 i k \sigma\left(U_{x}-U^{2}\right)+2 U^{3}-U_{x x}+\left[U_{x}, U\right] .
\end{aligned}
$$

Here, $q^{\dagger}$ refers to the Hermitian conjugate of a matrix $q$ and $*$ is the complex conjugation.

Let $e^{\sigma}:=\operatorname{diag}\left(e, e, e, e, e^{-1}\right)$. Introducing the matrix eigenfunction

$$
\mu(k ; x, t):=\psi(k ; x, t) e^{i k \sigma x+4 i k^{3} \sigma t},
$$

we rewrite (2.1) as

$$
\mu_{x}=-i k[\sigma, \mu]+U \mu,
$$

where $[\sigma, \mu]=\sigma \mu-\mu \sigma$. Let $\mu_{ \pm}=\left(\mu_{ \pm L}, \mu_{ \pm R}\right)$ be the matrix Jost solutions of (2.4) with the asymptotic conditions $\mu_{ \pm} \rightarrow I_{5 \times 5}$ as $x \rightarrow \pm \infty$ obtained from the Volterra integral equation

$$
\mu_{ \pm}(k ; x, t)=I_{5 \times 5}+\int_{ \pm \infty}^{x} e^{i k \sigma(\xi-x)} U(\xi, t) \mu_{ \pm}(k ; \xi, t) e^{-i k \sigma(\xi-x)} \mathrm{d} \xi
$$

Note that $\mu_{ \pm L}$ denotes each of the first four columns of $\mu_{ \pm}$and $\mu_{ \pm R}$ refers to the last column. It is easily seen that $\mu_{-L}$ and $\mu_{+R}$ are analytic in the upper complex half-plane $\mathbb{C}_{+}$and $\mu_{+L}$ 
and $\mu_{-R}$ are analytic in the lower complex half-plane $\mathbb{C}_{-}$. Since $\mu_{ \pm} e^{-i k \sigma x-4 i k^{3} \sigma t}$ are the solutions of the spectral problems (2.1) and (2.2), they are linearly related. Therefore, there is a scattering matrix $s(k)$ such that

$$
\mu_{-} e^{-i k \sigma x-4 i k^{3} \sigma t}=\mu_{+} e^{-i k \sigma x-4 i k^{3} \sigma t} s(k), \quad k \in \mathbb{R} .
$$

Noting that the matrix $U$ in (2.3) is traceless, one can show that $\operatorname{det} \mu_{ \pm}=1$. Combining it with (2.5) yields $\operatorname{det} s(k)=1$. We also note symmetry properties of $U$, viz.

$$
U^{\dagger}=-U, \quad \sigma_{1} U \sigma_{1}=U^{*}, \quad \sigma_{1}=\sigma_{1}^{-1}=\left(\begin{array}{ccccc}
0 & 1 & 0 & 0 & 0 \\
1 & 0 & 0 & 0 & 0 \\
0 & 0 & 0 & 1 & 0 \\
0 & 0 & 1 & 0 & 0 \\
0 & 0 & 0 & 0 & 1
\end{array}\right) .
$$

It follows from (2.4) that the Jost solutions $\mu_{ \pm}$have similar properties - viz.

$$
\mu_{ \pm}^{\dagger}\left(k^{*}\right)=\mu_{ \pm}^{-1}(k), \quad \sigma_{1} \mu_{ \pm}^{*}\left(-k^{*}\right) \sigma_{1}=\mu_{ \pm}(k)
$$

and using (2.5), we obtain

$$
s^{\dagger}\left(k^{*}\right)=s^{-1}(k), \quad \sigma_{1} s^{*}\left(-k^{*}\right) \sigma_{1}=s(k) .
$$

Consequently,

$$
\begin{array}{ll}
s_{22}^{\dagger}\left(k^{*}\right)=\operatorname{det}\left[s_{11}(k)\right], & s_{11}(k)=\sigma_{2} s_{11}^{*}\left(-k^{*}\right) \sigma_{2}, \\
s_{12}^{\dagger}\left(k^{*}\right)=-s_{21}(k) \operatorname{adj}\left[s_{11}(k)\right], & s_{21}^{*}\left(-k^{*}\right) \sigma_{2}=s_{21}(k), \\
\sigma_{2}=\sigma_{2}^{-1}=\left(\begin{array}{llll}
0 & 1 & 0 & 0 \\
1 & 0 & 0 & 0 \\
0 & 0 & 0 & 1 \\
0 & 0 & 1 & 0
\end{array}\right) &
\end{array}
$$

with adj $a$ denoting the adjoint matrix to $a$. Therefore, the scattering matrix $s(k)$ has the form

$$
s(k)=\left(\begin{array}{cc}
a(k) & -\operatorname{adj}\left[a^{\dagger}\left(k^{*}\right)\right] b^{\dagger}\left(k^{*}\right) \\
b(k) & \operatorname{det}\left[a^{\dagger}\left(k^{*}\right)\right]
\end{array}\right)
$$

where

$$
a(k)=\sigma_{2} a^{*}\left(-k^{*}\right) \sigma_{2}, \quad b^{*}\left(-k^{*}\right) \sigma_{2}=b(k) .
$$

If $t=0$ and $x \rightarrow+\infty$, the Eq. (2.5) yields

$$
\begin{aligned}
s(k) & =\lim _{x \rightarrow+\infty} e^{i k \sigma x} \mu_{-}(k ; x, 0) e^{-i k \sigma x} \\
& =I_{5 \times 5}+\int_{-\infty}^{+\infty} e^{i k \sigma \xi} U(\xi, 0) \mu_{-}(k ; \xi, 0) e^{-i k \sigma \xi} d \xi .
\end{aligned}
$$


It follows that

$$
\begin{aligned}
& a(k)=I_{4 \times 4}+\int_{-\infty}^{+\infty} q(\xi, 0) \mu_{-21}(k ; \xi, 0) d \xi \\
& b(k)=-\int_{-\infty}^{+\infty} e^{-2 i k \xi} q^{\dagger}(\xi, 0) \mu_{-11}(k ; \xi, 0) d \xi
\end{aligned}
$$

Set

$$
M(k ; x, t):= \begin{cases}\left(\mu_{-L}(k) a^{-1}(k), \mu_{+R}(k)\right), & k \in \mathbb{C}_{+}, \\ \left(\mu_{+L}(k), \frac{\mu_{-R}(k)}{\operatorname{det}\left[a^{\dagger}\left(k^{*}\right)\right]}\right), & k \in \mathbb{C}_{-} .\end{cases}
$$

Then formulas (2.5) and straightforward but tedious calculations show that for $k \in \mathbb{C} \backslash \mathbb{R}$, the matrix $M(k ; x, t)$ is the unique solution of the Riemann-Hilbert problem

$$
\begin{array}{ll}
M_{+}(k ; x, t)=M_{-}(k ; x, t) J(k ; x, t), & k \in \mathbb{R}, \\
M(k ; x, t) \rightarrow I_{5 \times 5}, & k \rightarrow \infty,
\end{array}
$$

where

$$
\begin{aligned}
& M_{ \pm}(k ; x, t)=\lim _{\varepsilon \rightarrow 0^{+}} M(k \pm i \varepsilon ; x, t), \\
& J(k ; x, t)=\left(\begin{array}{cc}
I_{4 \times 4}+\gamma^{\dagger}\left(k^{*}\right) \gamma(k) & e^{-2 i t \theta} \gamma^{\dagger}\left(k^{*}\right) \\
e^{2 i t \theta} \gamma(k) & 1
\end{array}\right), \\
& \theta(k ; x, t)=\frac{x}{t} k+4 k^{3}, \quad \gamma(k)=b(k) a^{-1}(k) .
\end{aligned}
$$

It is assumed that $a(k)$ is invertible and the reflection coefficient $\gamma(k)$, corresponding to the initial data $\left(u_{0}(x), v_{0}(x)\right)$, belongs to the Schwartz space $\mathscr{S}(\mathbb{R})$ and satisfies the conditions

$$
\gamma(k)=\gamma^{*}\left(-k^{*}\right) \sigma_{2}, \quad \sup _{k \in \mathbb{R}}|\gamma(k)|<\infty
$$

It is worth noting that since the jump matrix $J(k ; x, t)$ is positive definite, the RiemannHilbert problem (2.6) is uniquely solvable — cf. Vanishing Lemma in [1].

Theorem 2.1. If $M(k ; x, t), k \in \mathbb{C} \backslash \mathbb{R}$ is an analytic matrix-function satisfying the RiemannHilbert problem (2.6), then

$$
q(x, t)=\left(u(x, t), u^{*}(x, t), v(x, t), v^{*}(x, t)\right)^{\mathrm{T}}=2 i \lim _{k \rightarrow \infty}(k M(k ; x, t))_{12}
$$

is the solution of the Cauchy problem for the coupled Sasa-Satsuma equation (1.2).

Proof. Substituting the asymptotic expansion of $M(k ; x, t)$,

$$
M(k ; x, t)=I_{5 \times 5}+\frac{M_{1}(x, t)}{k}+\frac{M_{2}(x, t)}{k^{2}}+\mathscr{O}\left(k^{-3}\right), \quad k \rightarrow \infty,
$$


into (2.4) and comparing the coefficients of $\mathscr{O}(1 / k)$ gives (2.8).

The symmetry relation $\sigma_{1} J^{*}\left(-k^{*}\right) \sigma_{1}=J(k)$ for the jump matrix $J(k ; x, t)$ implies

$$
\sigma_{1} M^{*}\left(-k^{*}\right) \sigma_{1}=M(k) \text {. }
$$

Therefore, the expressions of $u(x, t)$ and $u^{*}(x, t), v(x, t)$ and $v^{*}(x, t)$ determined by (2.8) satisfy the corresponding conjugate relations.

\section{Long-Time Asymptotic Behavior}

\subsection{First transformation. Reoriented contour}

In order to solve the Riemann-Hilbert problem (2.6), we first establish a suitable factorisation of the jump matrix $J(k ; x, t)$. It is easily seen that $k_{0}=\sqrt{-x /(12 t)}$ are the stationary points of $\theta$, i.e. $\left.\frac{\partial \theta}{\partial k}\right|_{k= \pm k_{0}}=0$. Note that for $k \in\left(-\infty,-k_{0}\right) \cup\left(k_{0},+\infty\right)$, the jump matrix $J(k ; x, t)$ admits the upper/lower triangular factorisation

$$
J=\left(\begin{array}{cc}
I_{4 \times 4} & e^{-2 i t \theta} \gamma^{\dagger}\left(k^{*}\right) \\
0 & 1
\end{array}\right)\left(\begin{array}{cc}
I_{4 \times 4} & 0 \\
e^{2 i t \theta} \gamma(k) & 1
\end{array}\right),
$$

and for $k \in\left(-k_{0}, k_{0}\right)$, the lower/diagonal/upper triangular factorisation

$$
J=\left(\begin{array}{cc}
I_{4 \times 4} & 0 \\
\frac{e^{2 i t \theta} \gamma(k)}{1+\gamma(k) \gamma^{\dagger}\left(k^{*}\right)} & 1
\end{array}\right)\left(\begin{array}{cc}
I_{4 \times 4}+\gamma^{\dagger}\left(k^{*}\right) \gamma(k) & 0 \\
0 & \frac{1}{1+\gamma(k) \gamma^{\dagger}\left(k^{*}\right)}
\end{array}\right)\left(\begin{array}{cc}
I_{4 \times 4} & \frac{e^{-2 i t \theta} \gamma^{\dagger}\left(k^{*}\right)}{1+\gamma(k) \gamma^{\dagger}\left(k^{*}\right)} \\
0 & 1
\end{array}\right) .
$$

Considering a $4 \times 4$ matrix-function $\delta(k)$, which satisfies the following Riemann-Hilbert problem

$$
\begin{array}{ll}
\delta_{+}(k)=\delta_{-}(k)\left(I_{4 \times 4}+\gamma^{\dagger}\left(k^{*}\right) \gamma(k)\right), & k \in\left(-k_{0}, k_{0}\right), \\
\delta(k) \rightarrow I_{4 \times 4}, & k \rightarrow \infty,
\end{array}
$$

we arrive at the scalar Riemann-Hilbert problem

$$
\begin{array}{ll}
\operatorname{det} \delta_{+}(k)=\left(1+|\gamma(k)|^{2}\right) \operatorname{det} \delta_{-}(k), & k \in\left(-k_{0}, k_{0}\right), \\
\operatorname{det} \delta(k) \rightarrow 1, & k \rightarrow \infty .
\end{array}
$$

Since the jump matrix $I_{4 \times 4}+\gamma^{\dagger}\left(k^{*}\right) \gamma(k)$ is positive definite, the solution $\delta(k)$ is unique. By Plemelj formula [1], the Riemann-Hilbert problem (3.2) has the solution

$$
\operatorname{det} \delta(k)=\left(\frac{k-k_{0}}{k+k_{0}}\right)^{i v} e^{\chi(k)}
$$

where

$$
\begin{aligned}
& \nu=-\frac{1}{2 \pi} \log \left(1+\left|\gamma\left(k_{0}\right)\right|^{2}\right) \\
& \chi(k)=\frac{1}{2 \pi i} \int_{-k_{0}}^{k_{0}} \log \left(\frac{1+|\gamma(\xi)|^{2}}{1+\left|\gamma\left(k_{0}\right)\right|^{2}}\right) \frac{\mathrm{d} \xi}{\xi-k} .
\end{aligned}
$$


The uniqueness of the solution gives

$$
\delta(k)=\left(\delta^{\dagger}\left(k^{*}\right)\right)^{-1}=\sigma_{2} \delta\left(-k^{*}\right) \sigma_{2},
$$

and substituting (3.3) in (3.1) yields

$$
\begin{aligned}
\left|\delta_{+}(k)\right|^{2} & = \begin{cases}4+|\gamma(k)|^{2}, & k \in\left(-k_{0}, k_{0}\right), \\
4, & k \in\left(-\infty,-k_{0}\right) \cup\left(k_{0},+\infty\right),\end{cases} \\
\left|\delta_{-}(k)\right|^{2} & = \begin{cases}4-\frac{3|\gamma(k)|^{2}}{1+|\gamma(k)|^{2}}, & k \in\left(-k_{0}, k_{0}\right), \\
4, & k \in\left(-\infty,-k_{0}\right) \cup\left(k_{0},+\infty\right),\end{cases} \\
\left|\operatorname{det} \delta_{+}(k)\right|^{2} & = \begin{cases}1+|\gamma(k)|^{2}, & k \in\left(-k_{0}, k_{0}\right), \\
1, & k \in\left(-\infty,-k_{0}\right) \cup\left(k_{0},+\infty\right),\end{cases} \\
\left|\operatorname{det} \delta_{-}(k)\right|^{2} & = \begin{cases}\frac{1}{1+|\gamma(k)|^{2},} & k \in\left(-k_{0}, k_{0}\right), \\
1, & k \in\left(-\infty,-k_{0}\right) \cup\left(k_{0},+\infty\right) .\end{cases}
\end{aligned}
$$

Hence, by the maximum principle, we have

$$
|\delta(k)| \leqslant \text { const }<\infty, \quad|\operatorname{det} \delta(k)| \leqslant \text { const }<\infty, \quad k \in \mathbb{C} .
$$

Moreover, it follows from (3.3) that

$$
\left|\delta^{-1}(k)\right| \leqslant \text { const }<\infty, \quad\left|(\operatorname{det} \delta(k))^{-1}\right| \leqslant \text { const }<\infty, \quad k \in \mathbb{C} .
$$

We now set

$$
M^{\Delta}(k ; x, t):=M(k ; x, t) \Delta(k)
$$

where

$$
\Delta(k)=\left(\begin{array}{cc}
\delta^{-1}(k) & 0 \\
0 & \operatorname{det} \delta(k)
\end{array}\right)
$$

Changing the orientation for $k \in\left(-\infty,-k_{0}\right) \cup\left(k_{0},+\infty\right)$ as is shown in Fig. 1 and conjugating the Riemann-Hilbert problem (2.6), we obtain an equivalent Riemann-Hilbert problem - viz.

$$
\begin{array}{ll}
M_{+}^{\Delta}(k ; x, t)=M_{-}^{\Delta}(k ; x, t) J^{\Delta}(k ; x, t), & k \in \mathbb{R}, \\
M^{\Delta}(k ; x, t) \rightarrow I_{5 \times 5}, & k \rightarrow \infty,
\end{array}
$$

where the jump matrix $J^{\Delta}(k ; x, t)$ admits the lower-upper triangular factorisations:

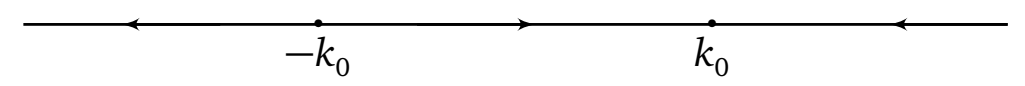

Figure 1: Reoriented contour. 
$J^{\Delta}(k ; x, t)=\left(\begin{array}{cc}I_{4 \times 4} & 0 \\ -\frac{e^{2 i t \theta} \gamma(k) \delta_{-}^{-1}(k)}{\operatorname{det} \delta_{-}(k)} & 1\end{array}\right)\left(\begin{array}{cc}I_{4 \times 4} & -e^{-2 i t \theta}\left[\operatorname{det} \delta_{+}(k)\right] \delta_{+}(k) \gamma^{\dagger}\left(k^{*}\right) \\ 0 & 1\end{array}\right)$,

$k \in\left(-\infty,-k_{0}\right) \cup\left(k_{0},+\infty\right)$,

$J^{\Delta}(k ; x, t)=\left(\begin{array}{cc}I_{4 \times 4} & 0 \\ \frac{e^{2 i t \theta} \gamma(k) \delta_{-}^{-1}(k)}{\left[1+\gamma(k) \gamma^{\dagger}\left(k^{*}\right)\right] \operatorname{det} \delta_{-}(k)} & 1\end{array}\right)\left(\begin{array}{cc}I_{4 \times 4} & \frac{e^{-2 i t \theta}\left[\operatorname{det} \delta_{+}(k)\right] \delta_{+}(k) \gamma^{\dagger}\left(k^{*}\right)}{1+\gamma(k) \gamma^{\dagger}\left(k^{*}\right)} \\ 0 & 1\end{array}\right)$,

$k \in\left(-k_{0}, k_{0}\right)$.

Introducing the vector-valued spectral function

$$
\rho(k)= \begin{cases}-\gamma^{\dagger}\left(k^{*}\right), & k \in\left(-\infty,-k_{0}\right) \cup\left(k_{0},+\infty\right), \\ \frac{\gamma^{\dagger}\left(k^{*}\right)}{1+\gamma(k) \gamma^{\dagger}\left(k^{*}\right)}, & k \in\left(-k_{0}, k_{0}\right),\end{cases}
$$

we can write the above defined function $J^{\Delta}(k ; x, t)$ as

$$
\begin{aligned}
& J^{\Delta}(k ; x, t) \\
& =\left(b_{-}\right)^{-1} b_{+}=\left(\begin{array}{cc}
I_{4 \times 4} & 0 \\
\frac{e^{2 i t \theta} \rho^{\dagger}\left(k^{*}\right) \delta_{-}^{-1}(k)}{\operatorname{det} \delta_{-}(k)} & 1
\end{array}\right)\left(\begin{array}{cc}
I_{4 \times 4} & e^{-2 i t \theta}\left[\operatorname{det} \delta_{+}(k)\right] \delta_{+}(k) \rho(k) \\
0 & 1
\end{array}\right), \quad k \in \mathbb{R} .
\end{aligned}
$$

\subsection{Second transformation. Equivalent Riemann-Hilbert problem}

In this subsection, we transform the Riemann-Hilbert problem (3.6) on $\mathbb{R}$ to an equivalent Riemann-Hilbert problem on the augmented contour

$$
\Sigma:=\mathbb{R} \cup L \cup L^{*}
$$

shown in Fig. 2 with $L$ defined by

$$
L:=\left\{k=k_{0}+k_{0} \alpha e^{3 \pi i / 4}:-\infty<\alpha \leqslant \sqrt{2}\right\} \cup\left\{k=-k_{0}+k_{0} \alpha e^{\pi i / 4}:-\infty<\alpha \leqslant \sqrt{2}\right\} .
$$

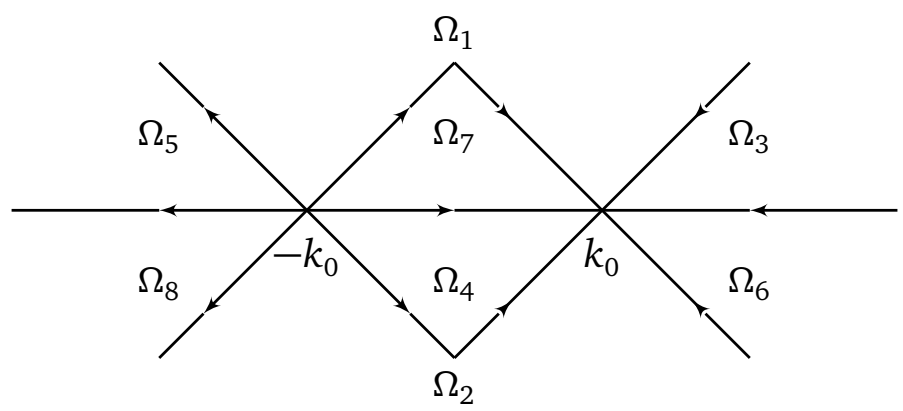

Figure 2: Oriented jump contour $\Sigma$. 
Besides, we also consider the contour

$$
L_{\epsilon}:=\left\{k=k_{0}+k_{0} \alpha e^{3 \pi i / 4}: \epsilon<\alpha \leqslant \sqrt{2}\right\} \cup\left\{k=-k_{0}+k_{0} \alpha e^{\pi i / 4}: \epsilon<\alpha \leqslant \sqrt{2}\right\}
$$

for $0<\epsilon<\sqrt{2}$.

In what follows, if there is a constant $C>0$ such that $|A| \leqslant C B$, then we will write $A \lesssim B$ and if, in such a constant $C$ depends on a parameter $\alpha$, then $A \lesssim_{\alpha} B$.

Theorem 3.1. The vector-valued spectral function $\rho(k)$ can be represented in the form

$$
\rho(k)=R(k)+h_{1}(k)+h_{2}(k), \quad k \in \mathbb{R},
$$

where $R(k)$ is piecewise rational, $h_{1}(k)$ analytic on $\mathbb{R}$, and $h_{2}(k)$ has an analytic continuation to L. If $0<k_{0}<C$, then for any positive integer $l$ and $t>0$, the following estimates hold:

$$
\begin{array}{ll}
\left|e^{-2 i t \theta(k)} h_{1}(k)\right| \lesssim \frac{1}{\left(1+|k|^{2}\right) t^{l}}, & k \in \mathbb{R}, \\
\left|e^{-2 i t \theta(k)} h_{2}(k)\right| \lesssim \frac{1}{\left(1+|k|^{2}\right) t^{l}}, & k \in L, \\
\left|e^{-2 i t \theta(k)} R(k)\right| \lesssim e^{-16 \epsilon^{2} k_{0}^{3} t}, \quad k \in L_{\epsilon} .
\end{array}
$$

Taking the Hermitian conjugate

$$
\rho^{\dagger}\left(k^{*}\right)=R^{\dagger}\left(k^{*}\right)+h_{1}^{\dagger}\left(k^{*}\right)+h_{2}^{\dagger}\left(k^{*}\right)
$$

leads to the same estimates for $e^{2 i t \theta(k)} h_{1}^{\dagger}\left(k^{*}\right), e^{2 i t \theta(k)} h_{2}^{\dagger}\left(k^{*}\right)$ and $e^{2 i t \theta(k)} R^{\dagger}\left(k^{*}\right)$ on $\mathbb{R} \cup L^{*}$.

Proof. It follows from [7, Proposition 1.92].

Factoring the matrices $b_{ \pm}$in (3.7) as

$$
\begin{aligned}
b_{+}= & b_{+}^{o} b_{+}^{a}=\left(I_{5 \times 5}+\omega_{+}^{o}\right)\left(I_{5 \times 5}+\omega_{+}^{a}\right)=\left(\begin{array}{cc}
I_{4 \times 4} & e^{-2 i t \theta}\left[\operatorname{det} \delta_{+}(k)\right] \delta_{+}(k) h_{1}(k) \\
0 & 1
\end{array}\right) \\
& \times\left(\begin{array}{cc}
I_{4 \times 4} & e^{-2 i t \theta}\left[\operatorname{det} \delta_{+}(k)\right] \delta_{+}(k)\left[h_{2}(k)+R(k)\right] \\
0 & 1
\end{array}\right), \\
b_{-}= & b_{-}^{o} b_{-}^{a}=\left(I_{5 \times 5}-\omega_{-}^{o}\right)\left(I_{5 \times 5}-\omega_{-}^{a}\right)=\left(\begin{array}{cc}
I_{4 \times 4} & 0 \\
-\frac{e^{2 i t \theta} h_{1}^{\dagger}\left(k^{*}\right) \delta_{-}^{-1}(k)}{\operatorname{det} \delta_{-}(k)} & 1
\end{array}\right) \\
& \times\left(\begin{array}{cc}
I_{4 \times 4} & 1
\end{array}\right),
\end{aligned}
$$

we set

$$
M^{\sharp}(k ; x, t)= \begin{cases}M^{\Delta}(k ; x, t), & k \in \Omega_{1} \cup \Omega_{2}, \\ M^{\Delta}(k ; x, t)\left(b_{-}^{a}\right)^{-1}, & k \in \Omega_{3} \cup \Omega_{4} \cup \Omega_{5}, \\ M^{\Delta}(k ; x, t)\left(b_{+}^{a}\right)^{-1}, & k \in \Omega_{6} \cup \Omega_{7} \cup \Omega_{8} .\end{cases}
$$


Straightforward computations show that $M^{\sharp}$ is the solution of the Riemann-Hilbert problem

$$
\begin{array}{ll}
M_{+}^{\sharp}(k ; x, t)=M_{-}^{\sharp}(k ; x, t) J^{\sharp}(k ; x, t), & k \in \Sigma, \\
M^{\sharp}(k ; x, t) \rightarrow I_{5 \times 5}, & k \rightarrow \infty,
\end{array}
$$

where

$$
J^{\sharp}(k ; x, t)=\left(b_{-}^{\sharp}\right)^{-1} b_{+}^{\sharp}= \begin{cases}I_{5 \times 5}^{-1} b_{+}^{a}, & k \in L, \\ \left(b_{-}^{a}\right)^{-1} I_{5 \times 5}, & k \in L^{*}, \\ \left(b_{-}^{o}\right)^{-1} b_{+}^{o}, & k \in \mathbb{R} .\end{cases}
$$

An asymptotic condition for $M^{\sharp}(k ; x, t)$ can be derived. For example, $\left(b_{+}^{a}\right)^{-1}$ converges to $I_{5 \times 5}$ as $k \rightarrow \infty$ in $\Omega_{6} \cup \Omega_{8}$. We first consider the domain $\Omega_{6}$. It follows from (3.4) that $\delta(k)$ and $\operatorname{det} \delta(k)$ are bounded for fixed $x, t$. This implies

$$
\left|e^{-2 i t \theta}[\operatorname{det} \delta(k)] \delta(k)\left[h_{2}(k)+R(k)\right]\right| \lesssim\left|e^{-2 i t \theta} h_{2}(k)\right|+\left|e^{-2 i t \theta} R(k)\right|, \quad \forall k \in \Omega_{6} .
$$

By the definition of $R(k)$, we have

$$
\left|e^{-2 i t \theta} R(k)\right| \lesssim \frac{\left|\sum_{j=0}^{m} \mu_{j}\left(k-k_{0}\right)^{j}\right|}{\left|(k-i)^{m+5}\right|} \lesssim \frac{1}{|k-i|^{5}}, \quad \forall k \in \Omega_{6} .
$$

Taking into account the convergence of $e^{-2 i t \theta} h_{2}(k)$, we finally obtain that $M^{\sharp}(k ; x, t) \rightarrow$ $I_{5 \times 5}$ as $k \rightarrow \infty$ in $\Omega_{6}$, and so on. The Riemann-Hilbert problem (3.9) is connected to singular integral equations as follows - cf. [7, P. 322] and [3]. We first define the following spaces

$$
\begin{aligned}
& \mathscr{L}^{p}(\Sigma)=\left\{f(k) \mid\left(\int_{\Sigma}|f(k)|^{p}|\mathrm{~d} k|\right)^{1 / p}<+\infty\right\}, \quad p \in\{1,2\} \\
& \mathscr{L}^{\infty}(\Sigma)=\left\{f(k)\left|\operatorname{esssup}_{k \in \Sigma}\right| f(k) \mid<+\infty\right\} .
\end{aligned}
$$

Set the Cauchy operators $C_{ \pm}$on $\Sigma$ by

$$
\left(C_{ \pm} f\right)(k)=\int_{\Sigma} \frac{f(\xi)}{\xi-k_{ \pm}} \frac{\mathrm{d} \xi}{2 \pi i}, \quad k \in \Sigma, \quad f \in \mathscr{L}^{2}(\Sigma),
$$

where $C_{+} f\left(C_{-} f\right)$ denotes the left (right) boundary value for the oriented contour $\Sigma$ in Fig. 2. For example, for $k>k_{0}$, we have

$$
\left(C_{+} f\right)(k)=\lim _{\varepsilon \rightarrow 0^{+}} \int_{\Sigma} \frac{f(\xi)}{\xi-(k-i \varepsilon)} \frac{\mathrm{d} \xi}{2 \pi i} .
$$

Moreover, the operators $C_{ \pm}$are bounded from $\mathscr{L}^{2}(\Sigma)$ to $\mathscr{L}^{2}(\Sigma)$, and $C_{+}-C_{-}=1$. Set

$$
\omega^{\sharp}=\omega_{+}^{\sharp}+\omega_{-}^{\sharp}, \quad \omega_{ \pm}^{\sharp}= \pm\left(b_{ \pm}^{\sharp}-I_{5 \times 5}\right) .
$$


Observe from Theorem 3.1 that, for fixed $x, t$, we then have

$$
\omega^{\sharp}, \omega_{ \pm}^{\sharp} \in \mathscr{L}^{1}(\Sigma) \cap \mathscr{L}^{\infty}(\Sigma) .
$$

Define

$$
C_{\omega^{\sharp}} f=C_{+}\left(f \omega_{-}^{\sharp}\right)+C_{-}\left(f \omega_{+}^{\sharp}\right)
$$

for a $5 \times 5$ matrix-valued function $f$. By property (3.10), $C_{\omega^{\sharp}}$ is a bounded map from $C_{\omega^{\sharp}}: \mathscr{L}^{2}(\Sigma)+\mathscr{L}^{\infty}(\Sigma) \rightarrow \mathscr{L}^{2}(\Sigma)$. If $\mu^{\sharp}(k ; x, t) \in \mathscr{L}^{2}(\Sigma)+\mathscr{L}^{\infty}(\Sigma)$ satisfies the singular integral equation

$$
\mu^{\sharp}=I_{5 \times 5}+C_{\omega} \mu^{\sharp} .
$$

Then

$$
M^{\sharp}(k ; x, t)=I_{5 \times 5}+\int_{\Sigma} \frac{\mu^{\sharp}(\xi ; x, t) \omega^{\sharp}(\xi ; x, t)}{\xi-k} \frac{\mathrm{d} \xi}{2 \pi i}, \quad k \in \mathbb{C} \backslash \Sigma
$$

is the solution of the Riemann-Hilbert problem (3.9).

Theorem 3.2. The solution $q(x, t)$ for the Cauchy problem of the coupled Sasa-Satsuma equation (1.2) has the form

$$
\begin{aligned}
q(x, t) & =\left(u(x, t), u^{*}(x, t), v(x, t), v^{*}(x, t)\right)^{\mathrm{T}} \\
& =-\frac{1}{\pi}\left(\int_{\Sigma}\left(\left(1-C_{\omega^{\sharp}}\right)^{-1} I_{5 \times 5}\right)(\xi) \omega^{\sharp}(\xi) \mathrm{d} \xi\right)_{12} .
\end{aligned}
$$

Proof. Using the Eqs. (2.8) and definition (3.5), (3.8) and (3.12), we obtain

$$
\begin{aligned}
q(x, t) & =\lim _{k \rightarrow \infty} 2 i\left(k M^{\sharp}(k ; x . t)\right)_{12} \\
& =\lim _{k \rightarrow \infty} 2 i\left(k M^{\Delta}(k ; x \cdot t)\right)_{12} \\
& =-\frac{1}{\pi}\left(\int_{\Sigma} \mu^{\sharp}(\xi ; x, t) \omega^{\sharp}(\xi) \mathrm{d} \xi\right)_{12} \\
& =-\frac{1}{\pi}\left(\int_{\Sigma}\left(\left(1-C_{\omega^{\sharp}}\right)^{-1} I_{5 \times 5}\right)(\xi) \omega^{\sharp}(\xi) \mathrm{d} \xi\right)_{12} .
\end{aligned}
$$

\subsection{Third transformation. Truncated contour}

Here we reduce the Riemann-Hilbert problem (3.9) on the contour $\Sigma$ to a RiemannHilbert problem on the truncated contour $\Sigma^{\prime}$, where $\Sigma^{\prime}=\Sigma \backslash\left(\mathbb{R} \cup L_{\epsilon} \cup L_{\epsilon}^{*}\right)$ shown in Fig. 3. Consider a function $\omega^{e}$ and write it in the form

$$
\omega^{e}=\omega^{a}+\omega^{b}+\omega^{c},
$$

where $\omega^{a}=\left.\omega^{\sharp}\right|_{\mathbb{R}}$ is supported on $\mathbb{R}$ and is composed of terms of type $h_{1}(k)$ and $h_{1}^{\dagger}\left(k^{*}\right)$ described in Theorem 3.1, the function $\omega^{b}=\left.\omega^{\sharp}\right|_{L \cup L^{*}}$ is supported on $L \cup L^{*}$ and is composed 


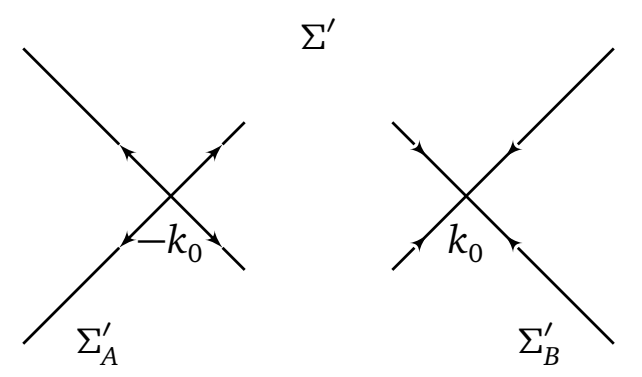

Figure 3: The oriented contour $\Sigma^{\prime}=\Sigma_{A}^{\prime} \cup \Sigma_{B}^{\prime}$.

of terms of type $h_{2}(k)$ and $h_{2}^{\dagger}\left(k^{*}\right)$, and $\omega^{c}=\left.\omega^{\sharp}\right|_{L_{\epsilon} \cup L_{\epsilon}^{*}}$ is supported on $L_{\epsilon} \cup L_{\epsilon}^{*}$ and is composed of terms of type $R(k)$ and $R^{\dagger}\left(k^{*}\right)$.

Define $\omega^{\prime}=\omega^{\sharp}-\omega^{e}$. It is easily seen that $\omega^{\prime}=0$ on $\Sigma \backslash \Sigma^{\prime}$. Hence, $\omega^{\prime}$ is supported on contour $\Sigma^{\prime}$ and is composed of terms of type $R(k)$ and $R^{\dagger}\left(k^{*}\right)$.

Lemma 3.1. If $\epsilon$ is sufficiently small, $0<k_{0}<C$ and $\tau=t k_{0}^{3}$, then

$$
\begin{aligned}
& \left\|\omega^{a}\right\|_{\mathscr{L}^{1}(\mathbb{R}) \cap \mathscr{L}^{2}(\mathbb{R}) \cap \mathscr{L}^{\infty}(\mathbb{R})} \lesssim t^{-l}, \\
& \left\|\omega^{b}\right\|_{\mathscr{L}^{1}\left(L \cup L^{*}\right) \cap \mathscr{L}^{2}\left(L \cup L^{*}\right) \cap \mathscr{L}^{\infty}\left(L \cup L^{*}\right)} \lesssim t^{-l}, \\
& \left\|\omega^{c}\right\|_{\mathscr{L}^{1}\left(L_{\epsilon} \cup L_{\epsilon}^{*}\right) \cap \mathscr{L}^{2}\left(L_{\epsilon} \cup L_{\epsilon}^{*}\right) \cap \mathscr{L}^{\infty}\left(L_{\epsilon} \cup L_{\epsilon}^{*}\right)} \lesssim e^{-16 \epsilon^{2} \tau}, \\
& \left\|\omega^{\prime}\right\|_{\mathscr{L}^{2}(\Sigma)} \lesssim \tau^{-1 / 4}, \quad\left\|\omega^{\prime}\right\|_{\mathscr{L}^{1}(\Sigma)} \lesssim \tau^{-1 / 2} .
\end{aligned}
$$

Proof. The proof of estimates (3.14)-(3.16) follows from Theorem 3.1. Indeed, for

$$
\left\{k=k_{0}+k_{0} \alpha e^{3 \pi i / 4}:-\infty<\alpha<\sqrt{2}\right\}
$$

the term $R(k)$ can be directly estimated as follows:

$$
|R(k)| \lesssim\left(1+|k|^{5}\right)^{-1} .
$$

Besides, since on this contour one has $\operatorname{Re}(i \theta) \geqslant 8 k_{0}^{3} \alpha^{2}$, the estimates (3.4) yield

$$
\left|e^{-2 i t \theta(k)}[\operatorname{det} \delta(k)] \delta(k) R(k)\right| \lesssim e^{-16 k_{0}^{3} \alpha^{2} t}\left(1+|k|^{5}\right)^{-1} .
$$

Analogously, considering $R^{\dagger}\left(k^{*}\right)$ on $L^{*}$, we obtain

$$
\left|e^{-2 i t \theta(k)}[\operatorname{det} \delta(k)]^{-1} R^{\dagger}\left(k^{*}\right) \delta^{-1}(k)\right| \lesssim e^{-16 k_{0}^{3} \alpha^{2} t}\left(1+|k|^{5}\right)^{-1}
$$

and direct calculations lead to (3.17).

Lemma 3.2. As $t \rightarrow \infty$, for $0<k_{0}<C$, the operator $\left(1-C_{\omega^{\prime}}\right)^{-1}: \mathscr{L}^{2}(\Sigma) \rightarrow \mathscr{L}^{2}(\Sigma)$ exists and is uniformly bounded - i.e.

$$
\left\|\left(1-C_{\omega^{\prime}}\right)^{-1}\right\|_{\mathscr{L}^{2}(\Sigma)} \lesssim 1 .
$$

The operator $\left(1-C_{\omega^{\sharp}}\right)^{-1}: \mathscr{L}^{2}(\Sigma) \rightarrow \mathscr{L}^{2}(\Sigma)$ also exists and is uniformly bounded - i.e.

$$
\left\|\left(1-C_{\omega^{\sharp}}\right)^{-1}\right\|_{\mathscr{L}^{2}(\Sigma)} \lesssim 1 .
$$


Proof. The conclusion follows [7, Proposition 2.23 and Corollary 2.25].

Theorem 3.3. We have

$$
\begin{aligned}
& \int_{\Sigma}\left(\left(1-C_{\omega^{\sharp}}\right)^{-1} I_{5 \times 5}\right)(\xi) \omega^{\sharp}(\xi) \mathrm{d} \xi \\
= & \int_{\Sigma}\left(\left(1-C_{\omega^{\prime}}\right)^{-1} I_{5 \times 5}\right)(\xi) \omega^{\prime}(\xi) \mathrm{d} \xi+\mathscr{O}\left(\tau^{-l}\right), \quad \tau \rightarrow \infty .
\end{aligned}
$$

Proof. Taking into account the second resolvent identity in [14], we write

$$
\begin{aligned}
& \left(\left(1-C_{\omega^{\sharp}}\right)^{-1} I_{5 \times 5}\right) \omega^{\sharp} \\
= & \left(\left(1-C_{\omega^{\prime}}\right)^{-1} I_{5 \times 5}\right) \omega^{\prime}+\omega^{e}+\left(\left(1-C_{\omega^{\prime}}\right)^{-1}\left(C_{\omega^{e}} I_{5 \times 5}\right)\right) \omega^{\sharp} \\
& +\left(\left(1-C_{\omega^{\prime}}\right)^{-1}\left(C_{\omega^{\prime}} I_{5 \times 5}\right)\right) \omega^{e}+\left(\left(1-C_{\omega^{\prime}}\right)^{-1} C_{\omega^{e}}\left(1-C_{\omega^{\sharp}}\right)^{-1}\right)\left(C_{\omega^{\sharp}} I_{5 \times 5}\right) \omega^{\sharp} .
\end{aligned}
$$

Consequently, Lemma 3.1 and Proposition 3.2 lead to the inequalities

$$
\begin{aligned}
& \left\|\omega^{e}\right\|_{\mathscr{L}^{1}(\Sigma)} \leqslant\left\|\omega^{a}\right\|_{\mathscr{L}^{1}(\mathbb{R})}+\left\|\omega^{b}\right\|_{\mathscr{L}^{1}\left(L \cup L^{*}\right)}+\left\|\omega^{c}\right\|_{\mathscr{L}^{1}\left(L_{\epsilon} \cup L_{\epsilon}^{*}\right)} \lesssim \tau^{-l}, \\
& \left\|\left(\left(1-C_{\omega^{\prime}}\right)^{-1}\left(C_{\omega^{e}} I_{5 \times 5}\right)\right) \omega^{\sharp}\right\|_{\mathscr{L}^{1}(\Sigma)} \\
\leqslant & \left\|\left(1-C_{\omega^{\prime}}\right)^{-1}\right\|_{\mathscr{L}^{2}(\Sigma)}\left\|C_{\omega^{e}} I_{5 \times 5}\right\|_{\mathscr{L}^{2}(\Sigma)}\left\|\omega^{\sharp}\right\|_{\mathscr{L}^{2}(\Sigma)} \lesssim\left\|\omega^{e}\right\|_{\mathscr{L}^{2}(\Sigma)}\left\|\omega^{\sharp}\right\|_{\mathscr{L}^{2}(\Sigma)} \lesssim \tau^{-l-1 / 4}, \\
& \left\|\left(\left(1-C_{\omega^{\prime}}\right)^{-1}\left(C_{\omega^{\prime}} I_{5 \times 5}\right)\right) \omega^{e}\right\|_{\mathscr{L}^{1}(\Sigma)} \\
\leqslant & \left\|\left(1-C_{\omega^{\prime}}^{-1}\right)\right\|_{\mathscr{L}^{2}(\Sigma)}\left\|C_{\omega^{\prime}} I_{5 \times 5}\right\|_{\mathscr{L}^{2}(\Sigma)}\left\|\omega^{e}\right\|_{\mathscr{L}^{2}(\Sigma)} \lesssim\left\|\omega^{\prime}\right\|_{\mathscr{L}^{2}(\Sigma)}\left\|\omega^{e}\right\|_{\mathscr{L}^{2}(\Sigma)} \lesssim \tau^{-l-1 / 4}, \\
& \|\left(\left(1-C_{\omega^{\prime}}\right)^{-1} C_{\omega^{e}}\left(1-C_{\omega^{\sharp}}\right)^{-1}\right)\left(C_{\left.\omega^{\sharp} I_{5 \times 5}\right) \omega^{\sharp}} \|_{\mathscr{L}^{1}(\Sigma)}\right. \\
\leqslant & \left\|\left(1-C_{\omega^{\prime}}\right)^{-1}\right\|_{\mathscr{L}^{2}(\Sigma)} \|\left(1-C_{\left.\omega^{\sharp}\right)^{-1}}\left\|_{\mathscr{L}^{2}(\Sigma)}\right\| C_{\omega^{e}}\left\|_{\mathscr{L}^{2}(\Sigma)}\right\| C_{\omega^{\sharp} I_{5 \times 5}}\left\|_{\mathscr{L}^{2}(\Sigma)}\right\| \omega^{\sharp} \|_{\mathscr{L}^{2}(\Sigma)}\right. \\
\lesssim & \left\|\omega^{e}\right\|_{\mathscr{L}^{\infty}(\Sigma)}\left\|\omega^{\sharp}\right\|_{\mathscr{L}^{2}(\Sigma)}^{2} \lesssim \tau^{-l-1 / 2} .
\end{aligned}
$$

Substituting these estimates into (3.19) gives (3.18).

Remark 3.1. Noting that $\omega^{\prime}(k)=0$ for $k \in \Sigma \backslash \Sigma^{\prime}$, we denote $\left.C_{\omega^{\prime}}\right|_{\mathscr{L}^{2}\left(\Sigma^{\prime}\right)}$ as the restriction of $C_{\omega^{\prime}}$ to $\mathscr{L}^{2}\left(\Sigma^{\prime}\right)$. For convenience, we rewrite $\left.C_{\omega^{\prime}}\right|_{\mathscr{L}^{2}\left(\Sigma^{\prime}\right)}$ as $C_{\omega^{\prime}}$, then

$$
\int_{\Sigma}\left(\left(1-C_{\omega^{\prime}}\right)^{-1} I_{5 \times 5}\right)(\xi) \omega^{\prime}(\xi) \mathrm{d} \xi=\int_{\Sigma^{\prime}}\left(\left(1-C_{\omega^{\prime}}\right)^{-1} I_{5 \times 5}\right)(\xi) \omega^{\prime}(\xi) \mathrm{d} \xi .
$$

Lemma 3.3. We have

$$
\begin{aligned}
q(x, t) & =\left(u(x, t), u^{*}(x, t), v(x, t), v^{*}(x, t)\right)^{\mathrm{T}} \\
& =-\frac{1}{\pi}\left(\int_{\Sigma^{\prime}}\left(\left(1-C_{\omega^{\prime}}\right)^{-1} I_{5 \times 5}\right)(\xi) \omega^{\prime}(\xi) \mathrm{d} \xi\right)_{12}+\mathscr{O}\left(\tau^{-l}\right), \quad \tau \rightarrow \infty .
\end{aligned}
$$


Proof. It follows directly from (3.13) and (3.18).

Consider the contour

$$
\Sigma^{\prime}=L^{\prime} \cup\left(L^{\prime}\right)^{*}
$$

where

$$
L^{\prime}=L \backslash L_{\epsilon},
$$

and let $\mu^{\prime}=\left(1-C_{\omega^{\prime}}\right)^{-1} I_{5 \times 5}$ on $\Sigma^{\prime}$. Similar to (3.12), we note that

$$
M^{\prime}(k ; x, t)=I_{5 \times 5}+\int_{\Sigma^{\prime}} \frac{\mu^{\prime}(\xi ; x, t) \omega^{\prime}(\xi ; x, t)}{\xi-k} \frac{\mathrm{d} \xi}{2 \pi i}
$$

solves the Riemann-Hilbert problem

$$
\begin{array}{ll}
M_{+}^{\prime}(k ; x, t)=M_{-}^{\prime}(k ; x, t) J^{\prime}(k ; x, t), & k \in \Sigma^{\prime}, \\
M^{\prime}(k ; x, t) \rightarrow I_{5 \times 5}, & k \rightarrow \infty,
\end{array}
$$

where

$$
\begin{aligned}
& J^{\prime}=\left(b_{-}^{\prime}\right)^{-1} b_{+}^{\prime}=\left(I_{5 \times 5}-\omega_{-}^{\prime}\right)^{-1}\left(I_{5 \times 5}+\omega_{+}^{\prime}\right), \\
& \omega^{\prime}=\omega_{+}^{\prime}+\omega_{-}^{\prime}, \\
& b_{+}^{\prime}=\left(\begin{array}{cc}
I_{4 \times 4} & e^{-2 i t \theta}[\operatorname{det} \delta(k)] \delta(k) R(k) \\
0 & 1
\end{array}\right), \quad b_{-}^{\prime}=I_{5 \times 5} \text { on } L^{\prime}, \\
& b_{+}^{\prime}=I_{5 \times 5}, \quad b_{-}^{\prime}=\left(\begin{array}{cc}
I_{4 \times 4} & 0 \\
-\frac{e^{2 i t \theta} R^{\dagger}\left(k^{*}\right) \delta^{-1}(k)}{\operatorname{det} \delta(k)} & 1
\end{array}\right) \text { on }\left(L^{\prime}\right)^{*} .
\end{aligned}
$$

\subsection{Fourth transformation. Decomposition of truncated contour}

In this subsection, we show how to separate the contributions of the two crosses in $\Sigma^{\prime}$ to the solution $q(x, t)$ in formula (3.20). Write $\Sigma^{\prime}=\Sigma_{A}^{\prime} \cup \Sigma_{B}^{\prime}$, where $\Sigma_{A}^{\prime}$ and $\Sigma_{B}^{\prime}$ are the disjoint crosses

$$
\begin{aligned}
& \Sigma_{A}^{\prime}=\left\{k=-k_{0}+h k_{0} e^{\pi i / 4} \mid-\infty<h \leqslant \epsilon\right\} \cup\left\{k=-k_{0}+h k_{0} e^{-\pi i / 4} \mid-\infty<h \leqslant \epsilon\right\}, \\
& \Sigma_{B}^{\prime}=\left\{k=k_{0}+h k_{0} e^{-3 \pi i / 4} \mid-\infty<h \leqslant \epsilon\right\} \cup\left\{k=k_{0}+h k_{0} e^{3 \pi i / 4} \mid-\infty<h \leqslant \epsilon\right\} .
\end{aligned}
$$

Set

$$
\omega_{ \pm}^{\prime}=\omega_{A \pm}^{\prime}+\omega_{B \pm}^{\prime}
$$

where

$$
\begin{array}{ll}
\omega_{A \pm}^{\prime}(k)=0, & k \in \Sigma_{B}^{\prime}, \\
\omega_{B \pm}^{\prime}(k)=0, & k \in \Sigma_{A}^{\prime},
\end{array}
$$

and define the operators $C_{\omega_{A}^{\prime}}$ and $C_{\omega_{B}^{\prime}}: \mathscr{L}^{2}\left(\Sigma^{\prime}\right)+\mathscr{L}^{\infty}\left(\Sigma^{\prime}\right) \rightarrow \mathscr{L}^{2}\left(\Sigma^{\prime}\right)$ similar to (3.11). Noting that $C_{\omega^{\prime}}=C_{\omega_{A}^{\prime}}+C_{\omega_{B}^{\prime}}$, we arrive at the following lemma. 


\section{Lemma 3.4.}

$$
\begin{aligned}
& \left\|C_{\omega_{B}^{\prime}} C_{\omega_{A}^{\prime}}\right\|_{\mathscr{L}^{2}\left(\Sigma^{\prime}\right)}=\left\|C_{\omega_{A}^{\prime}} C_{\omega_{B}^{\prime}}\right\|_{\mathscr{L}^{2}\left(\Sigma^{\prime}\right)} \lesssim_{k_{0}} \tau^{-1 / 2}, \\
& \left\|C_{\omega_{B}^{\prime}} C_{\omega_{A}^{\prime}}\right\|_{\mathscr{L} \infty\left(\Sigma^{\prime}\right) \rightarrow \mathscr{L}^{2}\left(\Sigma^{\prime}\right)}=\left\|C_{\omega_{A}^{\prime}} C_{\omega_{B}^{\prime}}\right\|_{\mathscr{L}^{\infty}\left(\Sigma^{\prime}\right) \rightarrow \mathscr{L}^{2}\left(\Sigma^{\prime}\right)} \lesssim_{k_{0}} \tau^{-3 / 4} .
\end{aligned}
$$

Proof. See Lemma 3.5 in Ref. [7].

Theorem 3.4. We have

$$
\begin{aligned}
q(x, t)= & \left(u(x, t), u^{*}(x, t), v(x, t), v^{*}(x, t)\right)^{\mathrm{T}} \\
= & -\frac{1}{\pi}\left(\int_{\Sigma^{\prime}}\left(\left(1-C_{\omega^{\prime}}\right)^{-1} I_{5 \times 5}\right)(\xi) \omega^{\prime}(\xi) d \xi\right)_{12} \\
= & -\frac{1}{\pi}\left(\int_{\Sigma_{A}^{\prime}}\left(\left(1-C_{\omega_{A}^{\prime}}\right)^{-1} I_{5 \times 5}\right)(\xi) \omega_{A}^{\prime}(\xi) d \xi\right)_{12} \\
& -\frac{1}{\pi}\left(\int_{\Sigma_{B}^{\prime}}\left(\left(1-C_{\omega_{B}^{\prime}}\right)^{-1} I_{5 \times 5}\right)(\xi) \omega_{B}^{\prime}(\xi) d \xi\right)_{12}+\mathscr{O}\left(\frac{c\left(k_{0}\right)}{\tau}\right), \quad \tau \rightarrow \infty .
\end{aligned}
$$

Proof. The representation

$$
\begin{aligned}
& \left(1-C_{\omega_{A}^{\prime}}-C_{\omega_{B}^{\prime}}\right)\left(1+C_{\omega_{A}^{\prime}}\left(1-C_{\omega_{A}^{\prime}}\right)^{-1}+C_{\omega_{B}^{\prime}}\left(1-C_{\omega_{B}^{\prime}}\right)^{-1}\right) \\
= & 1-C_{\omega_{B}^{\prime}} C_{\omega_{A}^{\prime}}\left(1-C_{\omega_{A}^{\prime}}\right)^{-1}-C_{\omega_{A}^{\prime}} C_{\omega_{B}^{\prime}}\left(1-C_{\omega_{B}^{\prime}}\right)^{-1}
\end{aligned}
$$

yields

$$
\begin{aligned}
\left(1-C_{\omega^{\prime}}\right)^{-1}= & 1+C_{\omega_{A}^{\prime}}\left(1-C_{\omega_{A}^{\prime}}\right)^{-1}+C_{\omega_{B}^{\prime}}\left(1-C_{\omega_{B}^{\prime}}\right)^{-1} \\
& +\left[1+C_{\omega_{A}^{\prime}}\left(1-C_{\omega_{A}^{\prime}}\right)^{-1}+C_{\omega_{B}^{\prime}}\left(1-C_{\omega_{B}^{\prime}}\right)^{-1}\right] \\
& \times\left[1-C_{\omega_{B}^{\prime}} C_{\omega_{A}^{\prime}}\left(1-C_{\omega_{A}^{\prime}}\right)^{-1}-C_{\omega_{A}^{\prime}} C_{\omega_{B}^{\prime}}\left(1-C_{\omega_{B}^{\prime}}\right)^{-1}\right]^{-1} \\
& \times\left[C_{\omega_{B}^{\prime}} C_{\omega_{A}^{\prime}}\left(1-C_{\omega_{A}^{\prime}}\right)^{-1}+C_{\omega_{A}^{\prime}} C_{\omega_{B}^{\prime}}\left(1-C_{\omega_{B}^{\prime}}\right)^{-1}\right] .
\end{aligned}
$$

This representation, combined with Lemmas 3.1, 3.4 and Proposition 3.2, gives (3.21).

\subsection{Rescaling and further reduction of Riemann-Hilbert problems}

Let us transform contours $\Sigma_{A}^{\prime}$ and $\Sigma_{B}^{\prime}$ to the crosses located at the origin. For this, we first extend the contours $\Sigma_{A}^{\prime}$ and $\Sigma_{B}^{\prime}$ as

$$
\begin{aligned}
& \hat{\Sigma}_{A}^{\prime}=\left\{-k_{0}+k_{0} \alpha e^{ \pm \pi i / 4} \mid \alpha \in \mathbb{R}\right\}, \\
& \hat{\Sigma}_{B}^{\prime}=\left\{k_{0}+k_{0} \alpha e^{ \pm \pi i / 4} \mid \alpha \in \mathbb{R}\right\},
\end{aligned}
$$


and define $\hat{\omega}_{A \pm}^{\prime}$ and $\hat{\omega}_{B \pm}^{\prime}$ on $\Sigma_{A}^{\prime}$ and $\Sigma_{B}^{\prime}$, respectively, by

$$
\hat{\omega}_{A \pm}^{\prime}=\left\{\begin{array}{ll}
\omega_{A \pm}^{\prime}, & k \in \Sigma_{A}^{\prime}, \\
0, & k \in \hat{\Sigma}_{A}^{\prime} \backslash \Sigma_{A}^{\prime},
\end{array} \quad \hat{\omega}_{B \pm}^{\prime}= \begin{cases}\omega_{B \pm}^{\prime}, & k \in \Sigma_{B}^{\prime}, \\
0, & k \in \hat{\Sigma}_{B}^{\prime} \backslash \Sigma_{B}^{\prime} .\end{cases}\right.
$$

Let $\Sigma_{A}$ and $\Sigma_{B}$ denote the contours $\left\{k=k_{0} \alpha e^{ \pm \pi i / 4} \mid \alpha \in \mathbb{R}\right\}$ in Fig. 4 oriented outward as in $\Sigma_{A}^{\prime}, \hat{\Sigma}_{A}^{\prime}$, and inward as in $\Sigma_{B}^{\prime}, \hat{\Sigma}_{B}^{\prime}$, respectively. Introduce the scaling operators

$$
\begin{aligned}
& N_{A}: \mathscr{L}^{2}\left(\hat{\Sigma}_{A}^{\prime}\right) \rightarrow \mathscr{L}^{2}\left(\Sigma_{A}\right), \quad f(k) \mapsto\left(N_{A} f\right)(k)=f\left(-k_{0}+\frac{k}{\sqrt{48 t k_{0}}}\right), \\
& N_{B}: \mathscr{L}^{2}\left(\hat{\Sigma}_{B}^{\prime}\right) \rightarrow \mathscr{L}^{2}\left(\Sigma_{B}\right), \quad f(k) \mapsto\left(N_{B} f\right)(k)=f\left(k_{0}+\frac{k}{\sqrt{48 t k_{0}}}\right),
\end{aligned}
$$

and define

$$
\omega_{A}=N_{A} \hat{\omega}_{A}^{\prime}, \quad \omega_{B}=N_{B} \hat{\omega}_{B}^{\prime} .
$$

Direct calculations show that

$$
C_{\hat{\omega}_{A}^{\prime}}=N_{A}^{-1} C_{\omega_{A}} N_{A}, \quad C_{\hat{\omega}_{B}^{\prime}}=N_{B}^{-1} C_{\omega_{B}} N_{B},
$$

where $C_{\omega_{A}}: \mathscr{L}^{2}\left(\Sigma_{A}\right) \rightarrow \mathscr{L}^{2}\left(\Sigma_{A}\right)$ and $C_{\omega_{B}}: \mathscr{L}^{2}\left(\Sigma_{B}\right) \rightarrow \mathscr{L}^{2}\left(\Sigma_{B}\right)$ are bounded operators. On the contour

$$
L_{A}=\left\{k=\alpha k_{0} \sqrt{48 t k_{0}} e^{-3 \pi i / 4}:-\epsilon<\alpha<+\infty\right\}
$$

we have

$$
\omega_{A}=\omega_{A+}=\left(\begin{array}{cc}
0 & \left(N_{A} s_{1}\right)(k) \\
0 & 0
\end{array}\right) .
$$

On the other hand, on the contour

$$
L_{A}^{*}=\left\{k=\alpha k_{0} \sqrt{48 t k_{0}} e^{3 \pi i / 4}:-\epsilon<\alpha<+\infty\right\}
$$

we have

$$
\omega_{A}=\omega_{A-}=\left(\begin{array}{cc}
0 & 0 \\
\left(N_{A} s_{2}\right)(k) & 0
\end{array}\right),
$$

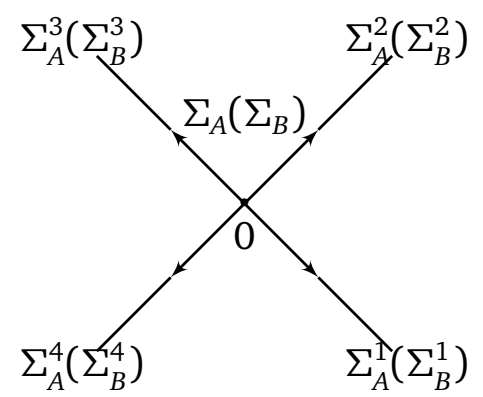

Figure 4: The oriented contour $\Sigma_{A}$ or $\Sigma_{B}$ (reoriented). 
where

$$
\begin{aligned}
& s_{1}(k)=e^{-2 i t \theta}[\operatorname{det} \delta(k)] \delta(k) R(k), \\
& s_{2}(k)=\frac{e^{2 i t \theta} R^{\dagger}\left(k^{*}\right) \delta^{-1}(k)}{\operatorname{det} \delta(k)} .
\end{aligned}
$$

Lemma 3.5. As $t \rightarrow \infty$, and $k \in L_{A}$, for an arbitrary positive integer $l$,

$$
\left|\left(N_{A} \tilde{\delta}\right)(k)\right| \lesssim t^{-l}
$$

where $\tilde{\delta}(k)=e^{-2 i t \theta}\left[\delta(k)-\operatorname{det} \delta(k) I_{4 \times 4}\right] R(k)$.

Proof. It follows from (3.1) and (3.2) that $\tilde{\delta}$ satisfies the following Riemann-Hilbert problem

$$
\begin{array}{ll}
\tilde{\delta}_{+}(k)=\tilde{\delta}_{-}(k)\left(1+|\gamma(k)|^{2}\right)+e^{-2 i t \theta} f(k), & k \in\left(-k_{0}, k_{0}\right), \\
\tilde{\delta}(k) \rightarrow 0, & k \rightarrow \infty,
\end{array}
$$

where $f(k)=\left[\delta_{-}\left(\gamma^{\dagger} \gamma-|\gamma|^{2} I_{4 \times 4}\right) R\right](k)$. The solution of this problem has the form

$$
\begin{aligned}
& \tilde{\delta}(k)=X(k) \int_{-k_{0}}^{k_{0}} \frac{e^{-2 i t \theta(\xi)} f(\xi)}{X_{+}(\xi)(\xi-k)} \frac{d \xi}{2 \pi i}, \\
& X(k)=\exp \left\{\frac{1}{2 \pi i} \int_{-k_{0}}^{k_{0}} \frac{\log \left(1+|\gamma(\xi)|^{2}\right)}{\xi-k} d \xi\right\} .
\end{aligned}
$$

Note that

$$
\begin{aligned}
\gamma^{\dagger} \gamma R-|\gamma|^{2} R & =\gamma^{\dagger} \gamma(R-\rho)-|\gamma|^{2}(R-\rho) \\
& =\left(|\gamma|^{2} I_{4 \times 4}-\gamma^{\dagger} \gamma\right)\left(h_{1}+h_{2}\right),
\end{aligned}
$$

and $\left(|\gamma|^{2} I_{4 \times 4}-\gamma^{\dagger} \gamma\right)$ consists of the components of $\gamma$. Therefore, following Theorem 3.1, we represent $f(k)$ as the sum $f(k)=f_{1}(k)+f_{2}(k)$, where $f_{2}(k)$ has an analytic continuation to $L_{t}$, cf. Fig. 5 , where

$$
\begin{aligned}
L_{t}:= & \left\{k=k_{0}+k_{0} \alpha e^{3 \pi i / 4}: 0 \leqslant \alpha \leqslant \sqrt{2}\left(1-\frac{1}{2 t}\right)\right\} \\
& \cup\left\{k=\frac{k_{0}}{t}-k_{0}+k_{0} \alpha e^{\pi i / 4}: 0 \leqslant \alpha \leqslant \sqrt{2}\left(1-\frac{1}{2 t}\right)\right\} .
\end{aligned}
$$

Then for $l \geqslant 2$, we have

$$
\begin{aligned}
& \left|e^{-2 i t \theta(k)} f_{1}(k)\right| \lesssim \frac{1}{\left(1+|k|^{2}\right) t^{l}}, \quad k \in \mathbb{R}, \\
& \left|e^{-2 i t \theta(k)} f_{2}(k)\right| \lesssim \frac{1}{\left(1+|k|^{2}\right) t^{l}}, \quad k \in L_{t} .
\end{aligned}
$$




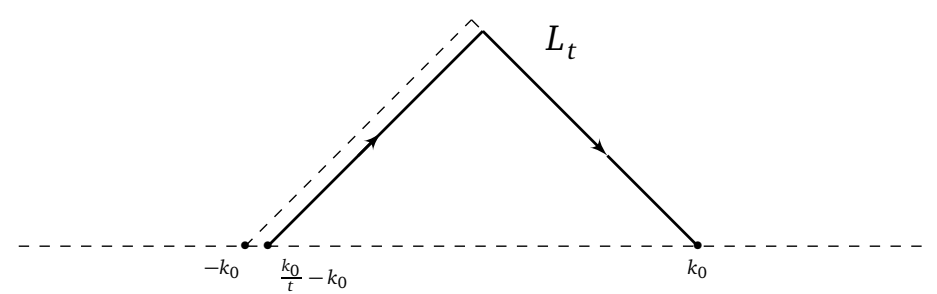

Figure 5: The contour $L_{t}$.

Since $k \in L_{A}$, it follows that

$$
\begin{aligned}
\left(N_{A} \tilde{\delta}\right)(k)= & X\left(\frac{k}{\sqrt{48 t k_{0}}}-k_{0}\right) \int_{-k_{0}}^{\frac{k_{0}}{t}-k_{0}} \frac{e^{-2 i t \theta(\xi)} f(\xi)}{X_{+}(\xi)\left(\xi+k_{0}-k / \sqrt{48 t k_{0}}\right)} \frac{d \xi}{2 \pi i} \\
& +X\left(\frac{k}{\sqrt{48 t k_{0}}}-k_{0}\right) \int_{\frac{k_{0}}{t}-k_{0}}^{k_{0}} \frac{e^{-2 i t \theta(\xi)} f_{1}(\xi)}{X_{+}(\xi)\left(\xi+k_{0}-k / \sqrt{48 t k_{0}}\right)} \frac{d \xi}{2 \pi i} \\
& +X\left(\frac{k}{\sqrt{48 t k_{0}}}-k_{0}\right) \int_{\frac{k_{0}}{t}-k_{0}}^{k_{0}} \frac{e^{-2 i t \theta(\xi)} f_{2}(\xi)}{X_{+}(\xi)\left(\xi+k_{0}-k / \sqrt{48 t k_{0}}\right)} \frac{d \xi}{2 \pi i} \\
:= & A_{1}+A_{2}+A_{3},
\end{aligned}
$$

and

$$
\begin{aligned}
& \left|A_{1}\right| \lesssim \int_{-k_{0}}^{\frac{k_{0}}{t}-k_{0}} \frac{\left|e^{-2 i t \theta(\xi)} f(\xi)\right|}{\left|\xi+k_{0}-k / \sqrt{48 t k_{0}}\right|} d \xi \lesssim t^{-l-1}, \\
& \left|A_{2}\right| \lesssim \int_{\frac{k_{0}}{t}-k_{0}}^{k_{0}} \frac{\left|e^{-2 i t \theta(\xi)} f_{1}(\xi)\right|}{\left|\xi+k_{0}-k / \sqrt{48 t k_{0}}\right|} d \xi \leqslant t^{-l} \frac{\sqrt{2} t}{k_{0}}\left(2 k_{0}-\frac{k_{0}}{t}\right) \lesssim t^{-l+1} .
\end{aligned}
$$

By using the Cauchy's theorem, we can evaluate the integral $A_{3}$ over the contour $L_{t}$ instead of the interval $\left(k_{0} / t-k_{0}, k_{0}\right)$ and obtain $\left|A_{3}\right| \lesssim t^{-l+1}$. Finally, we have (3.22).

Note. Similarly, if $t \rightarrow \infty$ and $k \in L_{A}^{*}$, then

$$
\left|\left(N_{A} \hat{\delta}\right)(k)\right| \lesssim t^{-l}
$$

where

$$
\hat{\delta}(k)=e^{2 i t \theta(k)} R^{\dagger}\left(k^{*}\right)\left[\delta^{-1}(k)-[\operatorname{det} \delta(k)]^{-1} I_{4 \times 4}\right] .
$$

Set

$$
\begin{aligned}
& J^{A^{0}}=\left(I_{5 \times 5}-\omega_{A^{0}-}\right)^{-1}\left(I_{5 \times 5}+\omega_{A^{0}+}\right), \\
& \delta_{A}=e^{\chi\left(-k_{0}\right)-8 i \tau}(192 \tau)^{i v / 2},
\end{aligned}
$$




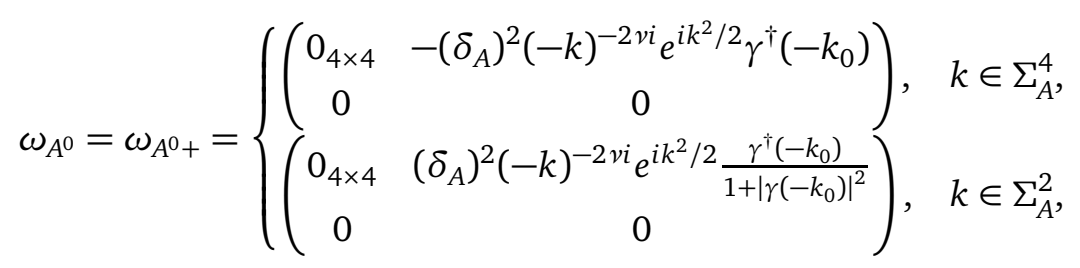

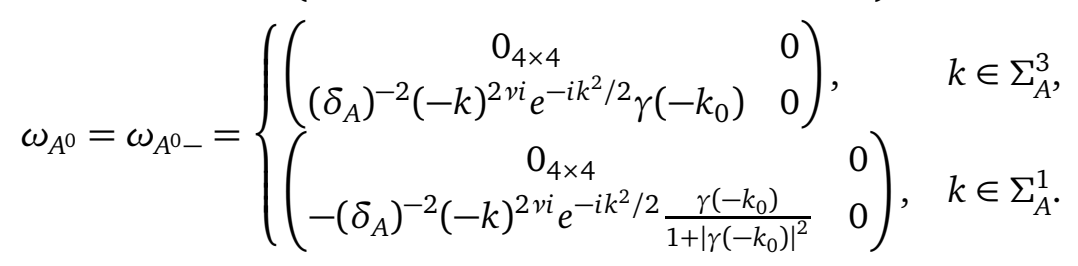

Lemma 3.5 and [7, Lemma 3.35] give

$$
\left\|\omega_{A}-\omega_{A^{0}}\right\|_{\mathscr{L}^{\infty}\left(\Sigma_{A}\right) \cap \mathscr{L}^{1}\left(\Sigma_{A}\right) \cap \mathscr{L}^{2}\left(\Sigma_{A}\right)} \lesssim_{k_{0}} t^{-1 / 2} \log t,
$$

and

$$
\begin{aligned}
& \int_{\Sigma_{A}^{\prime}}\left(\left(1-C_{\omega_{A}^{\prime}}\right)^{-1} I_{5 \times 5}\right)(\xi) \omega_{A}^{\prime}(\xi) d \xi \\
= & \int_{\hat{\Sigma}_{A}^{\prime}}\left(\left(1-C_{\hat{\omega}_{A}^{\prime}}\right)^{-1} I_{5 \times 5}\right)(\xi) \hat{\omega}_{A}^{\prime}(\xi) d \xi \\
= & \int_{\hat{\Sigma}_{A}^{\prime}}\left(N_{A}^{-1}\left(1-C_{\omega_{A}}\right)^{-1} N_{A} I_{5 \times 5}\right)(\xi) \hat{\omega}_{A}^{\prime}(\xi) d \xi \\
= & \int_{\hat{\Sigma}_{A}^{\prime}}\left(\left(1-C_{\omega_{A}}\right)^{-1} I_{5 \times 5}\right)\left(\left(\xi+k_{0}\right) \sqrt{48 t k_{0}}\right) N_{A} \hat{\omega}_{A}^{\prime}\left(\left(\xi+k_{0}\right) \sqrt{48 t k_{0}}\right) d \xi \\
= & \frac{1}{\sqrt{48 t k_{0}}} \int_{\Sigma_{A}}\left(\left(1-C_{\omega_{A}}\right)^{-1} I_{5 \times 5}\right)(\xi) \omega_{A}(\xi) d \xi \\
= & \frac{1}{\sqrt{48 t k_{0}}} \int_{\Sigma_{A}}\left(\left(1-C_{\omega_{A} 0}\right)^{-1} I_{5 \times 5}\right)(\xi) \omega_{A^{0}}(\xi) d \xi+\mathscr{O}\left(c\left(k_{0}\right) t^{-1} \log t\right) .
\end{aligned}
$$

The integral $\int_{\Sigma_{B}^{\prime}}\left(\left(1-C_{\omega_{B}^{\prime}}\right)^{-1} I_{5 \times 5}\right)(\xi) \omega_{B}^{\prime}(\xi) d \xi$ is evaluated analogously.

Theorem 3.5. We have

$$
\begin{aligned}
q(x, t)= & -\frac{1}{\sqrt{48 t k_{0}}}\left(\int_{\Sigma_{A}}\left(\left(1-C_{\omega_{A^{0}}}\right)^{-1} I_{5 \times 5}\right)(\xi) \omega_{A^{0}}(\xi) \frac{d \xi}{\pi}\right)_{12} \\
& -\frac{1}{\sqrt{48 t k_{0}}}\left(\int_{\Sigma_{B}}\left(\left(1-C_{\omega_{B} 0}\right)^{-1} I_{5 \times 5}\right)(\xi) \omega_{B^{0}}(\xi) \frac{d \xi}{\pi}\right)_{12} \\
& +\mathscr{O}\left(c\left(k_{0}\right) t^{-1} \log t\right), \quad \tau \rightarrow \infty .
\end{aligned}
$$


Proof. The relation (3.26) follows from Theorem 3.4 and the Eq. (3.25).

Let $k \in \mathbb{C} \backslash \Sigma_{A}$. Considering function

$$
M^{A^{0}}(k ; x, t)=I_{5 \times 5}+\int_{\Sigma_{A}} \frac{\left(\left(1-C_{\omega_{A} 0}\right)^{-1} I_{5 \times 5}\right)(\xi) \omega_{A^{0}}(\xi)}{\xi-k} \frac{d \xi}{2 \pi i},
$$

we note that it satisfies the Riemann-Hilbert problem

$$
\begin{array}{ll}
M_{+}^{A^{0}}(k ; x, t)=M_{-}^{A^{0}}(k ; x, t) J^{A^{0}}(k ; x, t), & k \in \Sigma_{A}, \\
M^{A^{0}}(k ; x, t) \rightarrow I_{5 \times 5}, & k \rightarrow \infty .
\end{array}
$$

In particular,

$$
M^{A^{0}}(k)=I_{5 \times 5}+\frac{M_{1}^{A^{0}}}{k}+\mathscr{O}\left(k^{-2}\right), \quad k \rightarrow \infty .
$$

It follows from (3.27) and (3.29) that

$$
M_{1}^{A^{0}}=-\int_{\Sigma_{A}}\left(\left(1-C_{\omega_{A^{0}}}\right)^{-1} I_{5 \times 5}\right)(\xi) \omega_{A^{0}}(\xi) \frac{d \xi}{2 \pi i} .
$$

There is a similar Riemann-Hilbert problem with $B^{0}$ on $\Sigma_{B}$, viz.

$$
\begin{array}{ll}
M_{+}^{B^{0}}(k ; x, t)=M_{-}^{B^{0}}(k ; x, t) J^{B^{0}}(k ; x, t), & k \in \Sigma_{B}, \\
M^{B^{0}}(k ; x, t) \rightarrow I_{5 \times 5}, & k \rightarrow \infty
\end{array}
$$

with

$$
\begin{aligned}
& J^{B^{0}}=\left(I_{5 \times 5}-\omega_{B^{0}}\right)^{-1}\left(I_{5 \times 5}+\omega_{B^{0}+}\right), \\
& \delta_{B}=e^{\chi\left(k_{0}\right)+8 i \tau}(192 \tau)^{-i v / 2},
\end{aligned}
$$

where

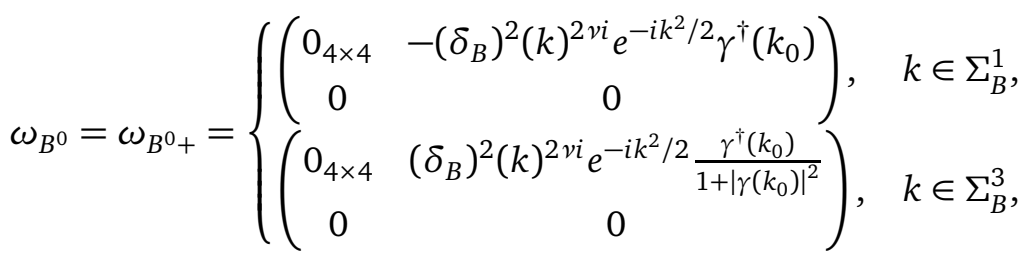

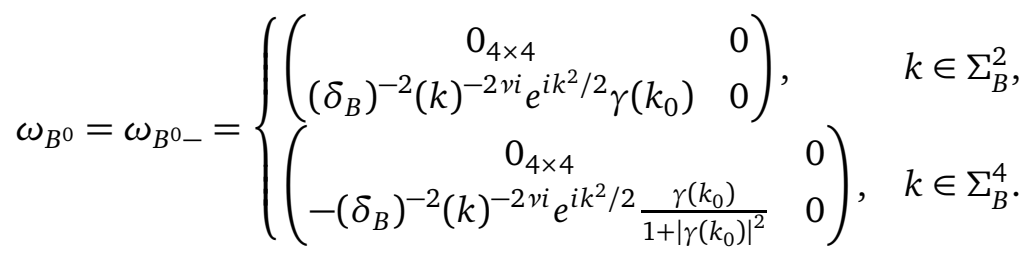

Combining (3.23)-(3.24) with (3.31)-(3.32) yields

$$
J^{A^{0}}(k)=\sigma_{1}\left(J^{B^{0}}\right)^{*}\left(-k^{*}\right) \sigma_{1} .
$$


By uniqueness,

$$
M^{A^{0}}(k)=\sigma_{1}\left(M^{B^{0}}\right)^{*}\left(-k^{*}\right) \sigma_{1}
$$

and, consequently,

$$
M_{1}^{A^{0}}=-\sigma_{1}\left(M_{1}^{B^{0}}\right)^{*} \sigma_{1} .
$$

Therefore, taking into account (3.26) and (3.30), we obtain

$$
q(x, t)=\frac{i}{\sqrt{12 t k_{0}}}\left(M_{1}^{A^{0}}-\sigma_{1}\left(M_{1}^{A^{0}}\right)^{*} \sigma_{1}\right)_{12}+\mathscr{O}\left(c\left(k_{0}\right) t^{-1} \log t\right) .
$$

\subsection{Solving a model problem}

Here, we are going to compute $\left(M_{1}^{A^{0}}\right)_{12}$. Define the matrix function

$$
\Psi(k)=H(k)(-k)^{-i v \sigma} e^{1 / 4 i k^{2} \sigma}, \quad H(k)=\left(\delta_{A}\right)^{-\sigma} M^{A^{0}}(k)\left(\delta_{A}\right)^{\sigma} .
$$

It follows from (3.28) that

$$
\Psi_{+}(k)=\Psi_{-}(k) v\left(-k_{0}\right), \quad k \in \Sigma_{A},
$$

where

$$
v\left(-k_{0}\right)=(-k)^{i v \sigma} e^{-1 / 4 i k^{2} \sigma}\left(\delta_{A}\right)^{-\sigma} J^{A^{0}}\left(\delta_{A}\right)^{\sigma} e^{1 / 4 i k^{2} \sigma}(-k)^{-i v \sigma} .
$$

Noting that on the rays $\Sigma_{A}^{1}, \Sigma_{A}^{2}, \Sigma_{A}^{3}, \Sigma_{A}^{4}$ the jump matrix $v\left(-k_{0}\right)$ does not depend on $k$, we write

$$
\frac{d \Psi_{+}(k)}{d k}=\frac{d \Psi_{-}(k)}{d k} v\left(-k_{0}\right) .
$$

The Eqs. (3.35) and (3.36) yield

$$
\frac{d \Psi_{+}(k)}{d k} \Psi_{+}^{-1}(k)=\frac{d \Psi_{-}(k)}{d k} \Psi_{-}^{-1}(k),
$$

so that $(d \Psi(k) / d k) \Psi^{-1}(k)$ has no jump across $\Sigma_{A}$ and is entire function. Besides, taking into account (3.34), we obtain

$$
\begin{aligned}
\frac{d \Psi(k)}{d k} \Psi^{-1}(k) & =\frac{d H(k)}{d k} H^{-1}(k)+\frac{1}{2} i k H(k) \sigma H^{-1}(k)-\frac{i v}{k} H(k) \sigma H^{-1}(k) \\
& =\mathscr{O}\left(\frac{1}{k}\right)+\frac{1}{2} i k \sigma-\frac{1}{2} i \delta_{A}^{-\sigma}\left[\sigma, M_{1}^{A^{0}}\right] \delta_{A}^{\sigma} .
\end{aligned}
$$

The Liouville's theorem yields

$$
\frac{d \Psi(k)}{d k}-\frac{1}{2} i k \sigma \Psi(k)=\beta \Psi(k),
$$

where

$$
\beta=-\frac{1}{2} i \delta_{A}^{-\sigma}\left[\sigma, M_{1}^{A^{0}}\right] \delta_{A}^{\sigma}=\left(\begin{array}{cc}
0 & \beta_{12} \\
\beta_{21} & 0
\end{array}\right)
$$


In particular

$$
\left(M_{1}^{A^{0}}\right)_{12}=i \delta_{A}^{2} \beta_{12} .
$$

Writing

$$
\Psi(k)=\left(\begin{array}{ll}
\Psi_{11}(k) & \Psi_{12}(k) \\
\Psi_{21}(k) & \Psi_{22}(k)
\end{array}\right)
$$

and using (3.37) gives

$$
\begin{aligned}
& \frac{d^{2} \beta_{21} \Psi_{11}(k)}{d k^{2}}=\left(\beta_{12} \beta_{21}+\frac{i}{2}-\frac{k^{2}}{4}\right) \beta_{21} \Psi_{11}(k), \\
& \Psi_{21}(k)=\frac{1}{\beta_{21} \beta_{12}}\left(\frac{d \beta_{21} \Psi_{11}(k)}{d k}-\frac{i}{2} k \beta_{21} \Psi_{11}(k)\right), \\
& \frac{d^{2} \Psi_{22}(k)}{d k^{2}}=\left(\beta_{21} \beta_{12}-\frac{i}{2}-\frac{k^{2}}{4}\right) \Psi_{22}(k), \\
& \beta_{21} \Psi_{12}(k)=\frac{d \Psi_{22}(k)}{d k}+\frac{i}{2} k \Psi_{22}(k) .
\end{aligned}
$$

It is well-known that the Weber equation

$$
\frac{d^{2} g(\zeta)}{d \zeta^{2}}+\left(a+\frac{1}{2}-\frac{\zeta^{2}}{4}\right) g(\zeta)=0
$$

has the solution

$$
g(\zeta)=c_{1} D_{a}(\zeta)+c_{2} D_{a}(-\zeta) .
$$

Here, $D_{a}$ is the standard parabolic-cylinder function. We recall that it satisfies the following equations

$$
\begin{aligned}
& \frac{d D_{a}(\zeta)}{d \zeta}+\frac{\zeta}{2} D_{a}(\zeta)-a D_{a-1}(\zeta)=0, \\
& D_{a}( \pm \zeta)=\frac{\Gamma(a+1) e^{i \pi a / 2}}{\sqrt{2 \pi}} D_{-a-1}( \pm i \zeta)+\frac{\Gamma(a+1) e^{-i \pi a / 2}}{\sqrt{2 \pi}} D_{-a-1}(\mp i \zeta)
\end{aligned}
$$

where $\Gamma$ denotes the Gamma function. Using [39, pp. 347-349] we write

$$
D_{a}(\zeta)= \begin{cases}\zeta^{a} e^{-\zeta^{2} / 4}\left(1+\mathscr{O}\left(\zeta^{-2}\right)\right), & |\arg \zeta|<\frac{3 \pi}{4}, \\ \zeta^{a} e^{-\zeta^{2} / 4}\left(1+\mathscr{O}\left(\zeta^{-2}\right)\right) & \frac{\pi}{4}<\arg \zeta<\frac{5 \pi}{4}, \\ -\frac{\sqrt{2 \pi}}{\Gamma(-a)} e^{a \pi i+\zeta^{2} / 4} \zeta^{-a-1}\left(1+\mathscr{O}\left(\zeta^{-2}\right)\right), & \\ \zeta^{a} e^{-\zeta^{2} / 4}\left(1+\mathscr{O}\left(\zeta^{-2}\right)\right) & -\frac{5 \pi}{4}<\arg \zeta<-\frac{\pi}{4} \\ -\frac{\sqrt{2 \pi}}{\Gamma(-a)} e^{-a \pi i+\zeta^{2} / 4} \zeta^{-a-1}\left(1+\mathscr{O}\left(\zeta^{-2}\right)\right), & \end{cases}
$$


as $\zeta \rightarrow \infty$. Set $a=-i \beta_{21} \beta_{12}$,

$$
\begin{aligned}
& \beta_{21} \Psi_{11}(k)=c_{1} D_{a}\left(e^{3 \pi i / 4} k\right)+c_{2} D_{a}\left(e^{-\pi i / 4} k\right), \\
& \Psi_{22}(k)=c_{3} D_{-a}\left(e^{-3 \pi i / 4} k\right)+c_{4} D_{-a}\left(e^{\pi i / 4} k\right) .
\end{aligned}
$$

If $\arg k \in(-\pi,-3 \pi / 4) \cup(3 \pi / 4, \pi)$ and $k \rightarrow \infty$, then

$$
\Psi_{11}(k)(-k)^{i v} e^{-i k^{2} / 4} \rightarrow I_{4 \times 4}, \quad \Psi_{22}(k)(-k)^{-i v} e^{i k^{2} / 4} \rightarrow 1 .
$$

It follows from (3.42) that

$$
\begin{aligned}
& \beta_{21} \Psi_{11}(k)=\beta_{21} e^{\pi v / 4} D_{a}\left(e^{3 \pi i / 4} k\right), \quad v=\beta_{21} \beta_{12}, \\
& \Psi_{22}(k)=e^{\pi v / 4} D_{-a}\left(e^{-3 \pi i / 4} k\right) .
\end{aligned}
$$

This and the Eqs. (3.39), (3.40) imply

$$
\begin{aligned}
& \Psi_{21}(k)=\beta_{21} e^{\pi(v+i) / 4} D_{a-1}\left(e^{3 \pi i / 4} k\right), \\
& \beta_{21} \Psi_{12}(k)=a e^{\pi(v+i) / 4} D_{-a-1}\left(e^{-3 \pi i / 4} k\right) .
\end{aligned}
$$

Analogously, if $\arg k \in(\pi / 4,3 \pi / 4)$ and $k \rightarrow \infty$, then

$$
\Psi_{11}(k)(-k)^{i v} e^{-i k^{2} / 4} \rightarrow I_{4 \times 4}, \quad \Psi_{22}(k)(-k)^{-i v} e^{i k^{2} / 4} \rightarrow 1,
$$

which implies

$$
\begin{aligned}
& \beta_{21} \Psi_{11}(k)=\beta_{21} e^{-3 \pi v / 4} D_{a}\left(e^{-\pi i / 4} k\right) \\
& \Psi_{22}(k)=e^{\pi v / 4} D_{-a}\left(e^{-3 \pi i / 4} k\right)
\end{aligned}
$$

Furthermore,

$$
\begin{aligned}
& \Psi_{21}(k)=\beta_{21} e^{-3 \pi(v+i) / 4} D_{a-1}\left(e^{-\pi i / 4} k\right), \\
& \beta_{21} \Psi_{12}(k)=a e^{\pi(\nu+i) / 4} D_{-a-1}\left(e^{-3 \pi i / 4} k\right) .
\end{aligned}
$$

In particular, if $\arg k=3 \pi / 4$, then

$$
\Psi_{+}(k)=\Psi_{-}(k)\left(\begin{array}{cc}
I_{4 \times 4} & 0 \\
-\gamma\left(-k_{0}\right) & 1
\end{array}\right) .
$$

Hence

$$
\begin{aligned}
& \beta_{21} e^{\pi(v+i) / 4} D_{a-1}\left(e^{3 \pi i / 4} k\right) \\
= & \beta_{21} e^{-3 \pi(v+i) / 4} D_{a-1}\left(e^{-\pi i / 4} k\right)-\gamma\left(-k_{0}\right) e^{\pi v / 4} D_{-a}\left(e^{-3 \pi i / 4} k\right) .
\end{aligned}
$$

We derive by (3.41) that

$$
\begin{aligned}
& D_{-a}\left(e^{-3 \pi i / 4} k\right) \\
= & \frac{\Gamma(-a+1) e^{-i \pi a / 2}}{\sqrt{2 \pi}} D_{a-1}\left(e^{-\pi i / 4} k\right)+\frac{\Gamma(-a+1) e^{i \pi a / 2}}{\sqrt{2 \pi}} D_{a-1}\left(e^{3 \pi i / 4} k\right) .
\end{aligned}
$$


Substituting (3.44) into (3.43) and separating the coefficients at the independent functions leads to the following equation:

$$
\beta_{21}=\frac{e^{\pi v / 2+3 \pi i / 4} \Gamma(-a+1)}{\sqrt{2 \pi}} \gamma\left(-k_{0}\right)=\frac{e^{\pi v / 2-3 \pi i / 4} \nu \Gamma(i v)}{\sqrt{2 \pi}} \gamma\left(-k_{0}\right) .
$$

Note that $\left(M^{A^{0}}\right)^{-1}(k)$ and $\left(M^{A^{0}}\right)^{\dagger}\left(k^{*}\right)$ satisfy the same Riemann-Hilbert problem and the uniqueness of the solution gives

$$
\left(M^{A^{0}}\right)^{-1}(k)=\left(M^{A^{0}}\right)^{\dagger}\left(k^{*}\right)
$$

Therefore,

$$
\beta_{12}=-\beta_{21}^{\dagger}=\frac{e^{\pi v / 2-\pi i / 4} v \Gamma(-i v)}{\sqrt{2 \pi}} \sigma_{2} \gamma^{\mathrm{T}}\left(k_{0}\right)
$$

Theorem 1.1 now follows from (3.33), (3.38) and (3.45).

\section{Acknowledgments}

This work is supported by the National Natural Science Foundation of China (Grant Nos. 11871440, 11931017).

\section{References}

[1] M.J. Ablowitz and A.S. Fokas, Complex Variables: Introduction and Applications, Cambridge University Press (2003).

[2] N. Akhmedieva, J.M. Soto-Crespo, N. Devine and N.P. Hoffmann, Rogue wave spectra of the Sasa-Satsuma equation, Phys. D 294, 37-42 (2015).

[3] R. Beals and R.R. Coifman, Scattering and inverse scattering for first order systems, Comm. Pure Appl. Math. 37, 39-90 (1984).

[4] P.J. Cheng, S. Venakides and X. Zhou, Long-time asymptotics for the pure radiation solution of the sine-Gordon equation, Commun. Partial. Differ. Equ. 24, 1195-1262 (1999).

[5] P. Deift, A.R. Its and X. Zhou, Long-time asymptotics for integrable nonlinear wave equations, in: Important Developments in Soliton Theory. Springer Series in Nonlinear Dynamics, A.S. Fokas and V.E. Zakharov (Eds), 181-204, Springer (1993).

[6] P.A. Deift and J. Park, Long-time asymptotics for solutions of the NLS equation with a delta potential and even initial data, Int. Math. Res. 24, 5505-5624 (2011).

[7] P. Deift and X. Zhou, A steepest descent method for oscillatory Riemann-Hilbert problems. Asymptotics for the MKdV equation, Ann. of Math. 137, 295-368 (1993).

[8] X.G. Geng, M.M. Chen and K.D. Wang, Long-time asymptotics of the coupled modified Kortewegde Vries equation, J. Geom. Phys. 142, 151-167 (2019).

[9] X.G. Geng, R.M. Li and B. Xue, A vector general nonlinear Schrödinger equation with $(m+n)$ components, J. Nonlinear Sci. 30, 991-1013 (2020).

[10] X.G. Geng and H. Liu, The nonlinear steepest descent method to long-time asymptotics of the coupled nonlinear Schrödinger equation, J. Nonlinear Sci. 28, 739-763 (2018).

[11] X.G. Geng, Y.Y. Zhai and H.H. Dai, Algebro-geometric solutions of the coupled modified Korteweg-de Vries hierarchy, Adv. Math. 263, 123-153 (2014). 
[12] C. Gilson, J. Hietarinta, J. Nimmo and Y. Ohta, Sasa-Satsuma higher-order nonlinear Schrödinger equation and its bilinearization and multisoliton solutions, Phys. Rev. E 68, 016614 (2003).

[13] K. Grunert and G. Teschl, Long-time asymptotics for the Korteweg-de Vries equation via nonlinear steepest descent, Math. Phys. Anal. Geom. 12, 287-324 (2009).

[14] E. Hille and R.S. Phillips, Functional Analysis and Semi-groups, AMS (1957).

[15] T. Kanna and K. Sakkaravarthi, Multicomponent coherently coupled and incoherently coupled solitons and their collisions, J. Phys. A 44, 285211 (2011).

[16] A.V. Kitaev and A.H. Vartanian, Leading-order temporal asymptotics of the modified nonlinear Schrödinger equation: solitonless sector, Inverse Probl. 13, 1311-1339 (1997).

[17] A.V. Kitaev and A.H. Vartanian, Asymptotics of solutions to the modified nonlinear Schrödinger equation: solution on a nonvanishing continuous background, SIAM J. Math. Anal. 30, 787-832 (1999).

[18] Y. Kodama, Optical solitons in a monomode fiber, J. Stat. Phys. 39, 597-614 (1985).

[19] Y. Kodama and A. Hasegawa, Nonlinear pulse propagation in a monomode dielectric guide, IEEE J. Quantum Elect 23, 510-524 (1987).

[20] R.M. Li and X.G. Geng, Rogue periodic waves of the sine-Gordon equation, Appl. Math. Lett. 102, 106147 (2020).

[21] R.M. Li and X.G. Geng, On a vector long wave-short wave-type model, Stud. Appl. Math. 144, 164-184 (2020).

[22] L.M. Ling, The algebraic representation for high order solution of Sasa-Satsuma equation, Discrete Contin. Dyn. Syst. Ser. S 9, 1975-2010 (2016).

[23] H. Liu, X.G. Geng and B. Xue, The Deift-Zhou steepest descent method to long-time asymptotics for the Sasa-Satsuma equation, J. Differ. Equ. 265, 5984-6008 (2018).

[24] X. Lü, Bright-soliton collisions with shape change by intensity redistribution for the coupled SasaSatsuma system in the optical fiber communications, Commun. Nonlinear Sci. Numer. Simul. 19, 3969-3987 (2014).

[25] W.X. Ma, Long-time asymptotics of a three-component coupled mKdV system, Mathematics. 7, 573 (2019).

[26] W.X. Ma, Long-time asymptotics of a three-component coupled nonlinear Schrödinger system, J. Geom. Phys. 153, 103669 (2020).

[27] W.X. Ma, Y. Zhang and Y.N. Tang, Symbolic computation of lump solutions to a combined equation involving three types of nonlinear terms, East Asian J. Appl. Math. 10, $732-745$ (2020).

[28] A. Mahalingam and K. Porsezian, Propagation of dark solitons in a system of coupled higherorder nonlinear Schrödinger equations, J. Phys. A 35, 3099-3109 (2002).

[29] A. Boutet de Monvel, A. Kostenko, D. Shepelsky and G. Teschl, Long-time asymptotics for the Camassa-Holm equation, SIAM J. Math. Anal. 41, 1559-1588 (2009).

[30] A. Boutet de Monvel, J. Lenells and D. Shepelsky, Long-time asymptotics for the DegasperisProcesi equation on the half-line, Ann. Inst. Fourier 69, 171-230 (2019).

[31] A. Boutet de Monvel and D. Shepelsky, A Riemann-Hilbert approach for the Degasperis-Procesi equation, Nonlinearity 26, 2081-2107 (2013).

[32] K. Nakkeeran, K. Porsezian, P.S. Sundaram and A. Mahalingam, Optical solitons in N-coupled higher order nonlinear Schrödinger equations, Phys. Rev. Lett. 80, 1425-1428 (1998).

[33] S. Nandy, Inverse scattering approach to coupled higher-order nonlinear Schrödinger equation and $N$-soliton solutions, Nuclear Phys. B 679, 647-659 (2004).

[34] N. Sasa and J. Satsuma, New-type of soliton solutions for a higher-order nonlinear Schrödinger equation, J. Phys. Soc. Jpn. 60, 409-417 (1991).

[35] A.H. Vartanian, Higher order asymptotics of the modified non-linear Schrödinger equation, Commun. Partial. Differ. Equ. 25, 1043-1098 (2000). 
[36] M.N. Vinoj and V.C. Kuriakose, Multisoliton solutions and integrability aspects of coupled higherorder nonlinear Schrödinger equations, Phys. Rev. E 62, 8719-8725 (2000).

[37] J. Wei, X.G. Geng and X. Zeng, The Riemann theta function solutions for the hierarchy of Bogoyavlensky lattices, Trans. Amer. Math. Soc. 371, 1483-1507 (2019).

[38] J. Wei, X. Wang and X.G. Geng, Periodic and rational solutions of the reduced Maxwell-Bloch equations, Commun. Nonlinear Sci. Numer. Simul. 59, 1-14 (2018).

[39] E.T. Whittaker and G.N. Watson, A Course of Modern Analysis, Cambridge University Press (1927).

[40] J.P. Wu and X.G. Geng, Inverse scattering transform of the coupled Sasa-Satsuma equation by Riemann-Hilbert approach, Commun. Theor. Phys. 67, 527-534 (2017).

[41] X. Wu, S.F. Tian and J.J. Yang, Riemann-Hilbert approach and $N$-soliton solutions for threecomponent coupled Hirota equations, East Asian J. Appl. Math. 10, 717-731 (2020).

[42] J. Xu and E.G. Fan, Long-time asymptotics for the Fokas-Lenells equation with decaying initial value problem: without solitons, J. Differ. Equ. 259, 1098-1148 (2015).

[43] J.K. Yang and D.J. Kaup, Squared eigenfunctions for the Sasa-Satsuma equation, J. Math. Phys. 50, 023504 (2009).

[44] Y.Y. Zhai and X.G. Geng, The coupled Sasa-Satsuma hierarchy: Trigonal curve and finite genus solutions, Anal. Appl. 15, 667-697 (2017).

[45] L.C. Zhao, Z.Y. Yang and L.M. Ling, Localized waves on continuous wave background in a twomode nonlinear fiber with high-order effects, J. Phys. Soc. Jpn. 83, 104401 (2014). 